\title{
Diffusion and Interdiffusion in Binary Metallic Melts
}

\author{
P. Kuhn, ${ }^{1}$ J. Horbach, ${ }^{2}$ F. Kargl, ${ }^{1}$ A. Meyer, ${ }^{1}$ and Th. Voigtmann ${ }^{1,3}$ \\ ${ }^{1}$ Institut für Materialphysik im Weltraum, Deutsches Zentrum für Luft- und Raumfahrt (DLR), 51170 Köln, Germany \\ ${ }^{2}$ Soft Matter Laboratory, IPKM, Heinrich-Heine Universität Düsseldorf, Universitätsstr. 1, 40225 Düsseldorf, Germany \\ ${ }^{3}$ Department of Physics, Heinrich-Heine Universität Düsseldorf, Universitätsstr. 1, 40225 Düsseldorf, Germany
}

(Dated: October 9, 2018)

\begin{abstract}
We discuss the dependence of self- and interdiffusion coefficients on temperature and composition for two prototypical binary metallic melts, Al-Ni and $\mathrm{Zr}-\mathrm{Ni}$, in molecular-dynamics (MD) computer simulations and the mode-coupling theory of the glass transition (MCT). Dynamical processes that are mainly entropic in origin slow down mass transport (as expressed through self diffusion) in the mixture as compared to the ideal-mixing contribution. Interdiffusion of chemical species is a competition of slow kinetic modes with a strong thermodynamic driving force that is caused by non-entropic interactions. The combination of both dynamic and thermodynamic effects causes qualitative differences in the concentration dependence of self-diffusion and interdiffusion coefficients. At high temperatures, the thermodynamic enhancement of interdiffusion prevails, while at low temperatures, kinetic effects dominate the concentration dependence, rationalized within MCT as the approach to its ideal-glass transition temperature $T_{c}$. The Darken equation relating self- and interdiffusion qualitatively reproduces the concentration-dependence in both Zr-Ni and Al-Ni, but quantitatively, the kinetic contributions to interdiffusion can be slower than the lower bound suggested by the Darken equation. As temperature is decreased, the agreement with Darken's equation improves, due to a strong coupling of all kinetic modes that is a generic feature predicted by MCT.
\end{abstract}

PACS numbers: 61.20.Ja 61.25.Mv 66.10.cg

\section{INTRODUCTION}

Mass transport processes in dense melts are governed by highly cooperative phenomena ${ }^{1}$. This makes physical modeling based on a priori information on their structural properties a challenge. In the hydrodynamic regime of long times and large spatial scales, these processes are characterized by a number of transport coefficients. In the case of binary mixtures, the self-diffusion coefficient $D_{\alpha}^{s}$ describes long-range transport of a tracer particle (of a given species $\alpha$ ); a single inter-diffusion coefficient $D_{c c}$ describes the decay of concentration fluctuations on large scales; the shear viscosity $\eta$ reflects the slow decay of microscopic stress fluctuations. These coefficients enter the mesoscopic and macroscopic modeling of materials; their values and parameter dependence remain important assumptions in these approaches 2 . Understanding mixing effects, i.e., the dependence of these coefficients on melt composition, is of utmost importance in designing materials, and reveals crucial information on the underlying microscopic physical mechanisms.

In this paper, we will investigate model binary metallic melts at various temperatures and composition by molecular-dynamics (MD) computer simulation. This technique allows to decompose the different contributions to mass transport,$\frac{\sqrt{3}}{}$ in particular to the inter-diffusion coefficient $D_{c c}=L \cdot \Phi$ that is composed of a purely kinetic (Onsager) coefficient $L$, and a thermodynamic factor $\Phi$. We will demonstrate that their composition dependence is qualitatively different, so that interdiffusion processes are described by a competition of two opposing forces, a dynamic and a thermodynamic one. The dynamic contribution can be understood as the precursor of kinetic arrest as described by the mode-coupling theory of the glass transition (MCT) ${ }^{4}$, and consequently depends sensitively on control parameters.

Reliable experimental data for the mass transport coefficients in metallic melts are scarce, despite their importance. Some self-diffusion coefficients are accessible in quasi-elastic neutron scattering $\sqrt{3 \sqrt{516}}$, but only for those atomic species that have a strong incoherent contribution to the low- $q$ scattering signal. Classical diffusioncouple experiments such as the long-capillary technique measure the species' concentration profile some time after the relaxation of a macroscopic concentration step, interpreted using the Fickian diffusion law ${ }^{7 / 8}$. In-situ methods, only recently developed, such as X-ray radiography $\sqrt{9}$ or neutron radiography $\sqrt{10}$ are required to ensure that the relevant transport mechanism that is probed in the experiment is indeed diffusion. Still, these measurements are plagued with experimental artefacts originating from free surfaces and/or inhomogeneities ${ }^{11}$. In addition, buoyancy-driven convective flow may necessitate experiments under micro-gravity conditions ${ }^{12}$. This prohibits the systematic exploration of the melt's state space encompassing temperature $T$ and number concentration of the species $x_{\alpha}$.

It is therefore tempting to establish relations between the different mass transport coefficients, to be able to infer the remaining ones from those that can be measured most reliably. One famous example is the "StokesEinstein" relation (first derived by Sutherland ${ }^{13}$ ) connecting the self-diffusion coefficient of a macroscopic Brownian particle to the shear viscosity of the suspending fluid, $D^{s} \sim k T / \eta$. Although valid only in the limit of infinitely large tracers (on the scale of molecular interac- 
tions in the fluid), the Stokes-Einstein relation has been reported to hold reasonably well for the self-diffusion of the fluid constituents themselves 14 . Recent careful measurements of self-diffusion in liquid $\mathrm{Zr}-\mathrm{Ni}$ suggest that $D^{s} \cdot \eta \sim$ const. holds in a large temperature range (without the $k T$ factor $)^{\sqrt{6}}$. This is interpreted as signalling that the mechanisms governing self-diffusion and viscosity, although at first sight rather different, are dominated by processes on the same time scale. Within MCT, these processes are identified with the slow structural relaxation of density fluctuations. Only for strongly supercooled glass formers, deviations are known in the form of a "fractional" Stokes-Einstein relation $D^{s} \sim \eta^{-\xi}$ (with $\xi<1)^{15 \mid 16}$, which is a remarkable deviation from the hydrodynamic origin of the original relation 17 . Despite the fortuitous character of the validity of the Stokes-Einstein relation applied to self-diffusion, it remains a useful concept for not-too-viscous melts, and forms the basis of microscopic rheological measurements in many complex liquids 18 .

A similar remarkable link between collective transport phenomena and tagged-particle motion is the so-called Darken equation $\frac{19}{19}$. It relates collective interdiffusion to the two self-diffusion coefficients. Labeling the species in a binary mixture by $\alpha=\mathrm{A}$ and $\mathrm{B}$, one can write

$$
\begin{aligned}
D_{c c}(x, T)=\left[x_{\mathrm{B}} D_{\mathrm{A}}^{s}(x, T)+\right. & \left.x_{\mathrm{A}} D_{\mathrm{B}}^{s}(x, T)\right] \times \\
& \times \Phi(x, T) \cdot S(x, T),
\end{aligned}
$$

where we denote by $x_{\alpha}$ the number concentration of either species, and use the symbol $x$ to denote a concentration dependence in cases where the particle label is irrelevant due to $\sum_{\alpha} x_{\alpha}=x_{\mathrm{A}}+x_{\mathrm{B}}=1$. The relation proposed by Darken on the basis of hydrodynamic arguments, corresponds to setting $S=1$. In other words, the Onsager kinetic coefficient $L$ is approximated as the weighted average of the species' self-diffusion coefficients, neglecting cross-correlation terms that arise in the collective process ${ }^{20}$. The thermodynamic factor $\Phi$ is connected to the second derivative of the Gibbs free energy $G$ w.r.t. the concentrations, equivalently written as the zero-wavenumber limit of the inverse of the concentration-fluctuation static structure factor $S_{c c}(q)$,

$$
\Phi(x, T)=\frac{x_{\mathrm{A}} x_{\mathrm{B}}}{k T} \frac{\partial^{2} G(x, T)}{\partial x_{\mathrm{A}} \partial x_{\mathrm{B}}}=\frac{x_{\mathrm{A}} x_{\mathrm{B}}}{S_{c c}(q=0 ; x, T)},
$$

where $S_{c c}(q)=x_{\mathrm{B}}^{2} S_{\mathrm{AA}}(q)+x_{\mathrm{A}}^{2} S_{\mathrm{BB}}(q)-2 x_{\mathrm{A}} x_{\mathrm{B}} S_{\mathrm{AB}}(q)$ in terms of the (Ashcroft-Langreth 21 ) partial static structure factors $S_{\alpha \beta}(q)$. It has been noted already in the discussion of the original contribution by Darken $\frac{19}{19}$ that Eq. (1) (with $S=1$ ) involves implicit assumptions, no excess volume upon mixing being one of them.

The Darken equation contains the correct limits for vanishing species concentration, i.e., $D_{c c} \rightarrow D_{\text {minority }}^{s}$ whenever $x_{\alpha} \rightarrow 0,1$ : the interdiffusion coefficient in the presence of a dilute concentration of one of the species (in binary mixtures) is given by the self-diffusion coefficient of this minority species. Note that in this limit, also $\Phi \rightarrow 1$ : For infinite dilution of one species, one expects this species to be randomly distributed in the embedding host system formed by the majority species. The Gibbs free energy then follows the ideal-mixing law, $G \sim x \ln x+(1-x) \ln (1-x)$, leading to $\Phi=1$. In a system that favors mixing, the intermediate-concentration minimum will typically be deeper, so that $\Phi>1$ results for finite $x$. This is the case for most dense metallic melts.

A notable feature of the Darken approximation is that it always estimates the Onsager coefficient $L$ to be bounded by the two self-diffusion coefficients, i.e., $\min \left(D_{\mathrm{A}}^{s}, D_{\mathrm{B}}^{s}\right) \leq L \leq \max \left(D_{\mathrm{A}}^{s}, D_{\mathrm{B}}^{s}\right)$. A "correction factor" $S$ is usually included in Eq. (1) to account for nonideal mixing effects and kinetic cross-correlations. In the case of chemical diffusion in crystals, it is called the Manning factor ${ }^{22}$. In the liquid, it can be expressed in terms of the distinct parts of the velocity autocorrelation functions $\frac{20}{2}$, and can thus be evaluated exactly from the MD simulation. We will use $S$ to quantify the extent to which the Darken approximation is valid or violated.

Mode-coupling theory establishes a structuredynamics relationship based on the equilibrium liquid-state static structure factors ${ }^{4}$. In principle, given an effective interaction potential for some metallic melt, and following some ad-hoc approximations, the theory is able to calculate dynamical features in the viscous melt. It predicts transport coefficients such as viscosity, self-diffusivity, or interdiffusion, based on the assumption that the slow relaxation typical for a viscous fluid is governed by a slow decay of density fluctuations. MCT describes the effect that in dense liquids, particles are transiently trapped in nearest-neighbor cages. Transport out of these cages is possible by highly collective processes induced by thermal fluctions, and these become ever more ineffective when the density is increased or temperature is lowered. Eventually, this mechanism described by MCT arrests at an ideal fluid-to-glass transition at some critical temperature $T_{c}$. Approaching $T_{c}$ from above, diffusion coefficients and inverse viscosity diminish much more quickly than expected from an Arrhenius temperature dependence. For temperatures $T<T_{c}$ (where also strong deviations from the Stokes-Einstein relation are seen), other transport mechanisms dominate that have been described as solid-like 1 123124; these are not the aim of our discussion here. They typically cause Arrhenius-like behavior with widely different activation energies between the species 525 .

Arguably the simplest model system for dense mixtures is the hard-sphere system. Here one incorporates only the core repulsion between atoms, modeled as an infinite energy barrier that keeps particles separated by at least their hard-core diameter $d$. All other interaction forces are set to zero. Consequently, temperature does not change the thermodynamic state of the system, as there is no intrinsic energy scale. The dynamics of the hard-sphere system becomes slow if density is increased much like it slows down upon cooling in ordinary melts. 
Hence, density and inverse temperature are often used interchangably as a rule-of-thumb to discuss qualitative features of slow relaxation. The appropriate measure for the number density of a hard-sphere system is the dimensionless packing fraction, i.e., the fraction of the sample volume filled by the hard spheres. This accounts for a trivial change in number density when comparing systems with different particle sizes. The hard-sphere system has the additional advantage, that a parameter-free, first-principles approximation for the static structure factors exists in analytic form, namely the Percus-Yevick (PY) structure factor ${ }^{27}$. The predictions of MCT based on the PY approximation for binary hard-sphere mixtures have been established in great detail in the regime relevant for metallic melts 28 31.

Based on the hard-sphere analogy, MCT suggests that the kinetic coefficients at constant temperature display a minimum at intermediate $x$, while the viscosity should display a similar maximum. This arises, because for binary mixtures of spheres with size ratio $\delta \gtrsim 0.75$ and close to unity, the increased disorder in the cages favors glass formation at constant volume fraction 29 . For smaller size ratio, glass formation becomes suppressed upon mixing, but this regime is not relevant for metallic melts if one estimates effective hard-core sizes by, e.g., covalent radii. Translating the hard-sphere result into a change of the glass-transition temperature, the transition line $T_{c}(x)$ can be expected to exhibit a maximum at intermediate $x$. The maximum will occur in the vicinity of $x=1 / 2$, but the precise location will depend on the size ratio 29 .

In real metallic melts, the scenario is complicated by the fact that the pure components will have different glass-transition temperatures (would they not crystallize). Hence, the expected minimum in kinetic coefficients can be superimposed by an "ideal-mixing" trend that is either monotonically increasing or decreasing. In principle, MCT can predict the qualitative behavior of transport coefficients based on experimental data for the equilibrium static structure. However, this requires knowledge of all partial static structure factors $S_{\alpha \beta}(q)$ over a reasonably large range of wave numbers $q$. This is not easily achieved, in metallic melts although in principle possible, say, with neutron scattering where isotope substitution allows to vary the relative scattering lengths of the constituents. Three independent measurements then give all three elements of the symmetric $2 \times 2$ matrix $\boldsymbol{S}(q)$ in the binary mixture, onto which MCT calculations can be based. This was so far carried out for a particular $\mathrm{Zr}-\mathrm{Ni}$ composition at a single temperature ${ }^{32}$.

The hard-sphere model serves as a reference to quantify purely entropic effects of the dynamics. In realistic melts, non-entropic contributions to the interactions result in stronger chemical short-range order. The latter has been shown to change, e.g., the ratio of transport coefficients 32: in a binary $\mathrm{Zr}-\mathrm{Ni}$ melt, based on entropic contributions one expects $\mathrm{Ni}$ diffusion to be faster than $\mathrm{Zr}$ diffusion due to the smaller size of $\mathrm{Ni}$ atoms. Yet, taking into account within MCT the strong $\mathrm{Zr}-\mathrm{Ni}$ interactions that modify the partial static structure factors, the theory predicts both diffusion coefficients to be virtually identical, at least for the considered composition and temperature. This qualitative change induced by non-entropic interactions asks for a classification of various metallic melts according to the relation between their transport coefficients (such as hard-sphere like or non-hard-sphere like $e^{5}$ ).

In this paper, we compare MD simulation predictions for the mass transport coefficients of two exemplary binary metallic melts, $\mathrm{Al}-\mathrm{Ni}$ and $\mathrm{Zr}-\mathrm{Ni}$, and their composition dependence. These are model systems where experimental investigations in the molten state are possible; first results are available $\mathrm{e}^{20 \mid 32} \sqrt[35]{35}$, with further measurements continuing to date. We also use the static structure factors obtained by our MD simulations as input to the MCT equations of motion, in order to compare the theory's predictions for these specific melts with the dynamical results obtained in the simulation. Doing so, we are able to identify the observed trends of the mass transport coefficients upon mixing with some of MCT's generic predictions.

The comparison of $\mathrm{Al}-\mathrm{Ni}$ and $\mathrm{Zr}-\mathrm{Ni}$ allows to test which transport mechanisms are generic; both systems, while sharing one atomic species, show very different glassforming ability, and very different phase diagrams. Dynamically, they represent systems with a relatively weak effective size difference $\left(\delta_{\mathrm{Al}-\mathrm{Ni}} \approx 0.87\right)$, respectively a relatively strong one $\left(\delta_{\mathrm{Zr}-\mathrm{Ni}} \approx 0.78\right)$.

The MD simulations are performed with recent embedded-atom potentials that have been proven to be good models in terms of reproducing the melt's characteristics with purely classical molecular dynamics. It should be stressed that this modeling is intrinsically approximate. We therefore do not claim our results to be quantitative predictions for real $\mathrm{Al}-\mathrm{Ni}$ or $\mathrm{Zi}-\mathrm{Ni}$ melts, but rather for model systems that allow to understand the relevant mass transport mechanisms in such melts qualitatively and semi-quantitatively.

This paper is organized as follows: we will recollect the details of the MD simulations and mode-coupling theory analysis in Sec. II. Results are discussed in Sec. III first for the $\mathrm{Al}-\mathrm{Ni} \mathrm{MD}$ simulations, then for the $\mathrm{Zr}-\mathrm{Ni}$ simulations, and finally for the mode-coupling calculations based on the MD-simulated static structure factors for both systems. Finally, Sec. IV contains some conclusions.

\section{METHODS}

\section{A. MD Simulation}

Molecular-dynamics (MD) simulations were carried out using effective interaction potentials of the embedded atom type (EAM). One writes the total potential 
energy of a binary system as

$$
U=\frac{1}{2} \sum_{i, j \neq i} V_{\alpha(i) \beta(j)}\left(r_{i j}\right)+\sum_{i} F_{\alpha(i)}\left(\bar{\rho}_{i}\right),
$$

where $\alpha(i)$ denotes the species to which particle number $i$ belongs. $V_{\alpha \beta}(r)$ is the pair interaction potential between species $\alpha$ and $\beta$, assumed to depend on the distance between the particles, $r_{i j}=\left|\vec{r}_{i}-\vec{r}_{j}\right|$, only. The embedding energy $F_{\alpha(i)}$ of species $\alpha$ at site $\vec{r}_{i}$ is given by the electron density $\bar{\rho}_{i}$ from all other atoms. The latter is written as

$$
\bar{\rho}_{i}=\sum_{j \neq i} \rho_{\alpha(j)}\left(r_{i j}\right)
$$

the sum over the electron densities at site $\vec{r}_{i}$ due to a particle of species $\alpha$ at site $\vec{r}_{j}$. The exact functional forms of the pair potential $V_{\alpha \beta}(r)$, of the electron densities $\rho_{\alpha}(r)$, and of the embedding energies $F_{\alpha}(\rho)$ are empirical choices usually based on ab initio simulations and crystallographic data.

For Al-Ni, a potential proposed by Mishin et al! was used, as in earlier work of some of the present authors $\$ 20 / 33 / 37 / 38$. We only summarize the main aspects of the simulation here, and refer to these publications for details. MD simulations were performed by equilibrating the starting configurations in the NVT ensemble (with $N=1500$ particles), with a temperature-dependent volume corresponding to the average at a given pressure $p$, as determined from $N p T$ Monte Carlo simulations. After that, microcanonical MD simulations were performed to extract the dynamical quantities. Simulation runs covered more than $10^{5}$ time steps using the velocityVerlet integrator with time steps varying between $1 \mathrm{fs}$ $(T \geq 1500 \mathrm{~K})$ and $2.5 \mathrm{fs}$ (lower $T$ ). At each temperature, $T=1795 \mathrm{~K}, 1500 \mathrm{~K}, 1250 \mathrm{~K}, 1000 \mathrm{~K}$, and $900 \mathrm{~K}$, eight runs with independent initial configurations were averaged over to improve statistics. Compositions were chosen as $x_{\mathrm{Al}}=0.1,0.25,0.4,0.5,0.7,0.8$, and 0.9 at the highest temperature. At lower temperatures data were only obtained for $x_{\mathrm{Al}}=0.1,0.25,0.4$, and 0.8 . The self-diffusion and inter-diffusion coefficients are obtained via the appropriate Einstein relations from the long-time behavior of the mean-squared displacement and the corresponding interdiffusion quantity, see Ref. 20 for details.

The potential for $\mathrm{Zr}-\mathrm{Ni}$ was originally developed by

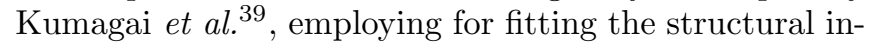
formation of amorphous $\mathrm{Zr}_{70} \mathrm{Ni}_{30}$, and mainly lattice parameters of the crystalline state. To improve the potential for the molten state we are interested in, we have adjusted the parametrization of Ref. 39 to reproduce quantitatively experimental Ni-diffusion data 20 . As further information, static structure factors of $\mathrm{Zr}_{64} \mathrm{Ni}_{36}$ were taken into account ${ }^{32}$; here, perfect agreement could not be enforced since a strong pre-peak emerges in the static structure factor of the melt that is much weaker in the MD simulation. Compared to Ref. 39, the potential energy was scaled by an overall factor $\alpha=1.86$, and the parameter $f_{e, \mathrm{ZrNi}}$, describing the electron-density ratio of atomic species $\mathrm{Zr}$ to $\mathrm{Ni}$, was changed from the value $f_{e}=0.215$ determinde by Kumagai et al., to $f_{e}=0.79$. For details on the parameters entering the $\mathrm{Zr}-\mathrm{Ni}$ potential, we refer to Ref. 39 .

The glass-transition dynamics in Zr-Ni melts has been studied previously by MD simulation combined with an analysis in terms of MCT by Teichler and coworkers $41+44$. The effective MD potential employed in these and related studies differs from the one we use here. We will remark on relevant differences below, where appropriate.

For the $\mathrm{Zr}-\mathrm{Ni}$ system, simulations were run at $x_{\mathrm{Zr}}=$ $0.36,0.5$, and 0.64 over a temperature window covering the onset of slow dynamics and about two orders of magnitude of slowing down. At $T=1400 \mathrm{~K}$, additional compositions $x_{\mathrm{Zr}}=0.2,0.43,0.57$, and 0.8 were considered. To discuss isothermic concentration dependences, the results for the different compositions obtained from runs at different temperatures $T<1400 \mathrm{~K}$ were interpolated linearly to constant $T$.

\section{B. Mode-Coupling Theory}

A recent monograph ${ }^{4}$ describes the mode-coupling theory of the glass transition and its application to experiments on molecular and hard-sphere-like colloidal glass formers in detail. MCT calculations based on MDsimulated partial static structure factors have been performed before (see Ref. 45 for an early example). Details for the present application follow those presented earlier for the $\mathrm{Al}_{80} \mathrm{Ni}_{20}$ simulation 38 .

MCT assumes that the slow dynamics of a dense melt is governed by the relaxation of density fluctuations. The statistics of the latter are encoded in the partial dynamic structure factors, or collective intermediate scattering functions to wave vector $\vec{q}$,

$$
S_{\alpha \beta}(q, t)=\frac{1}{N} \sum_{k_{\alpha}=1}^{N_{\alpha}} \sum_{l_{\beta}=1}^{N_{\beta}}\left\langle\exp \left[i \vec{q} \cdot\left[\vec{r}_{k_{\alpha}}(t)-\vec{r}_{l_{\beta}}(0)\right]\right\rangle,\right.
$$

where $\vec{r}_{k_{\alpha}}(t)$ marks the position of the particle labeled $k_{\alpha}$ at time $t$. At $t=0$ these functions yield the partial static structure factors $S_{\alpha \beta}(q)$. For an isotropic, translationally invariant equilibrium system, the dynamic structure factors depend on the wave vectors only through its magnitude $q=|\vec{q}|$, and on the time difference between the two density fluctuations. Following a projection operator scheme, an equation of motion is derived for the matrix $\boldsymbol{S}(q, t)$ (see Ref. 29),

$$
\begin{aligned}
\boldsymbol{J}^{-1}(q) \partial_{t}^{2} \boldsymbol{S}(q, t)+\boldsymbol{S}^{-1}(q) \cdot \boldsymbol{S}(q, t) \\
+\int_{0}^{t} \boldsymbol{M}\left(q, t-t^{\prime}\right) \cdot \partial_{t^{\prime}} \boldsymbol{S}\left(q, t^{\prime}\right) d t^{\prime}=\mathbf{0}
\end{aligned}
$$

The matrix $J_{\alpha \beta}(q)=q^{2} v_{\mathrm{th}, \alpha}^{2} \delta_{\alpha \beta}=q^{2} k_{B} T / m_{\alpha} \delta_{\alpha \beta}$ sets the thermal velocities governing the short-time relaxation. The long-time relaxation is dominated by 
retarded-friction effects that arise from slow collective dynamics. In the MCT approximation, they are captured through a memory kernel that is a nonlinear functional of the density correlation functions,

$$
\begin{array}{r}
M_{\alpha \beta}(q, t)=\frac{1}{2 q^{2}} \frac{n}{x_{\alpha} x_{\beta}} \int \frac{d^{3} k}{(2 \pi)^{3}} \sum_{\alpha^{\prime} \beta^{\prime} \alpha^{\prime \prime} \beta^{\prime \prime}} V_{\alpha \alpha^{\prime} \alpha^{\prime \prime}}(\vec{q}, \vec{k}) \times \\
V_{\beta \beta^{\prime} \beta^{\prime \prime}}(\vec{q}, \vec{k}) S_{\alpha^{\prime} \beta^{\prime}}(k, t) S_{\alpha^{\prime \prime} \beta^{\prime \prime}}(p, t)
\end{array}
$$

with $p=|\vec{q}-\vec{k}|$ and $n$ the total number density. For the coupling vertices we get $V_{\alpha \alpha^{\prime} \alpha^{\prime \prime}}(\vec{q}, \vec{k})=(\vec{q}$. $\vec{k} / q) c_{\alpha \alpha^{\prime}}(k) \delta_{\alpha \alpha^{\prime \prime}}+(\vec{q} \cdot \vec{p} / q) c_{\alpha \alpha^{\prime \prime}}(p) \delta_{\alpha \alpha^{\prime}}$ after neglecting a part that depends on the (unknown) static triplet correlation function. In this approximation, the static-structure factor matrix alone, through the related Ornstein-Zernike direct correlation function $c_{\alpha \beta}(q)=(1 / n)\left(\delta_{\alpha \beta} / x_{\alpha}-\right.$ $\left.\left(S^{-1}\right)_{\alpha \beta}\right)$, is sufficient to fully determine the MCT equations of motion.

To calculate self-diffusion coefficients, one needs to characterize the tracer-particle dynamics in the dense melt. This is achieved using the self-part of the intermediate scattering function,

$$
\phi_{\alpha}^{s}(q, t)=\frac{1}{N_{\alpha}} \sum_{k=1}^{N_{\alpha}}\left\langle\exp \left[i \vec{q} \cdot\left[\vec{r}_{k}(t)-\vec{r}_{k}(0)\right]\right\rangle .\right.
$$

The equation of motion for this tagged-particle density correlation function is similar to its collective counterpart,

$$
\begin{aligned}
& \frac{1}{q^{2} v_{\mathrm{th}, \alpha}^{2}} \partial_{t}^{2} \phi_{\alpha}^{s}(q, t)+\phi_{\alpha}^{s}(q, t) \\
& \quad+\int_{0}^{t} M_{\alpha}^{s}\left(q, t-t^{\prime}\right) \partial_{t^{\prime}} \phi_{\alpha}^{s}\left(q, t^{\prime}\right) d t^{\prime}=0 .
\end{aligned}
$$

Here, the retarded-friction memory kernel is given, in the MCT approximation, by a combination of the hostsystem density fluctuations and those of the tagged particle, hence

$$
\begin{array}{r}
M_{\alpha}^{s}(q, t)=\frac{n}{q^{2}} \int \frac{d^{3} k}{(2 \pi)^{3}} \sum_{\alpha^{\prime} \beta^{\prime}}(\vec{q} \cdot \vec{k} / q)^{2} c_{\alpha \alpha^{\prime}}(k) c_{\alpha \beta^{\prime}}(k) \times \\
S_{\alpha^{\prime} \beta^{\prime}}(k, t) \phi_{\alpha}^{s}(p, t)
\end{array}
$$

In the $q \rightarrow 0$ limit, the tagged-particle density correlation function is related to the mean-squared displacement $(\mathrm{MSD}) \delta r_{\alpha}^{2}(t)$ by $\phi_{\alpha}^{s}(q, t)=1-q^{2} \delta r_{\alpha}^{2}(t) / 6+\mathcal{O}\left(q^{4}\right)$. One readily verifies that for the MSD, an equation similar to Eq. 9) holds,

$$
\partial_{t} \delta r_{\alpha}^{2}(t)+v_{\mathrm{th}, \alpha}^{2} \int_{0}^{t} m_{\alpha}^{s}\left(t-t^{\prime}\right) \delta r_{\alpha}^{2}\left(t^{\prime}\right) d t^{\prime}=6 v_{\mathrm{th}, s}^{2} t,
$$

with $m_{\alpha}^{s}(t)=\lim _{q \rightarrow 0} q^{2} M_{\alpha}^{s}(q, t)$. The diffusion coefficient follows from $\delta r_{\alpha}^{2}(t \rightarrow \infty) \sim 6 D_{\alpha} t$, and is explicitly calculated as

$$
D_{\alpha}=\frac{1}{\int_{0}^{\infty} m_{\alpha}^{s}(t) d t}
$$

In a multi-component system, the memory kernels $M_{\alpha \beta}(q, t)$ display a $1 / q^{2}$ divergence for $q \rightarrow 0$, which is connected with the fact that particle numbers are conserved, but momentum of the individual species is not conserved. As a result, one obtains the hydrodynamic modes that are connected to interdiffusion. Writing $N_{\alpha \beta}(t)=\lim _{q \rightarrow 0} q^{2} M_{\alpha \beta}(q, t)$, we obtain

$$
N_{\alpha \beta}(t)=\frac{1}{x_{\alpha} x_{\beta}} \int d k \sum_{\alpha^{\prime} \beta^{\prime}} V_{\alpha \beta \alpha^{\prime} \beta^{\prime}}^{0}(k) \Delta S_{\alpha \beta \alpha^{\prime} \beta^{\prime}}(k, t)
$$

with $\Delta S_{\alpha \beta \alpha^{\prime} \beta^{\prime}}=S_{\alpha \beta} S_{\alpha^{\prime} \beta^{\prime}}-S_{\alpha^{\prime} \beta} S_{\alpha \beta^{\prime}}$ and $V_{\alpha \beta \alpha^{\prime} \beta^{\prime}}^{0}=$ $\left(n /\left(6 \pi^{2}\right)\right) k^{4} c_{\alpha \alpha^{\prime}}(k) c_{\beta \beta^{\prime}}(k)$. Specifically for binary mixtures,

$$
N_{\alpha \beta}(t)=\frac{(-1)^{\alpha+\beta}}{x_{\alpha} x_{\beta}} \int d k V_{\mathrm{AABB}}^{0}(k) \operatorname{det} \boldsymbol{S}(k, t) .
$$

This encodes the symmetries of the interdiffusion process and highlights that in a binary mixture, only one independent interdiffusion mode appears in the low- $q$ dynamics of density fluctuations.

The interdiffusion coefficient $D_{c c}$ is obtained from the concentration fluctuations $\varrho_{c}=x_{\mathrm{B}} \varrho_{\mathrm{A}}-x_{\mathrm{A}} \varrho_{\mathrm{B}}$, as

$$
D_{c c}=\frac{1}{S_{c c}(0) \int_{0}^{\infty} m_{c c}(t) d t}
$$

where $m_{c c}(t)=N_{\mathrm{AA}}(t) / x_{\mathrm{B}}^{2}$. For $x \rightarrow 0$ or $x \rightarrow 1$, the MCT expression for $D_{c c}$, Eq. (15) reduces to the one for the self-diffusion coefficient of the minority species, Eq. 12): if, say, $x_{\mathrm{B}} \rightarrow 0$, there holds $S_{\mathrm{BB}}(k, t) \sim$ $x_{\mathrm{B}} \phi_{\mathrm{B}}^{s}(k, t)$ and $\operatorname{det} \boldsymbol{S}(k, t) / x_{\mathrm{B}} \rightarrow S_{\mathrm{AA}}(k, t) \phi_{\mathrm{B}}^{s}(k, t)$, so that the memory kernel $x_{\mathrm{B}} N_{\mathrm{BB}}(t)$ in Eq. (14) becomes identical to the memory kernel $m_{\mathrm{B}}^{s}(t)$ in Eq. (11).

At the glass transition, MCT predicts that all memory kernels become non-decaying functions of time if they couple sufficiently strongly to collective density fluctuations. Hence, the integral in the denominators of Eq. (12) and (15) diverge, so that $D_{c c}, D_{\alpha} \rightarrow 0$ as one approaches the glass transition from the liquid side. In the present context, we do not discuss the possibility of weak coupling of one species, which may cause the corresponding diffusion coefficient to be non-zero in the glass $46 / 47$. This possibility makes clear that self- and interdiffusion are governed by tagged-particle respectively collective density fluctuations that are in principle different aspects of the dynamics, and might in fact decouple. However, in typical glass-forming melts above and close to $T_{c}$, the coupling between these relaxation modes is so strong, that approximations connecting one to the other may hold rather well.

In writing the MCT equations of motion as above, we have tacitly neglected short-time contributions to the memory kernels that are expected to be subdominant close to the MCT glass transition. These include a damping term that is responsible for the behavior of the diffusion coefficients in the less dense liquid, and is the object 


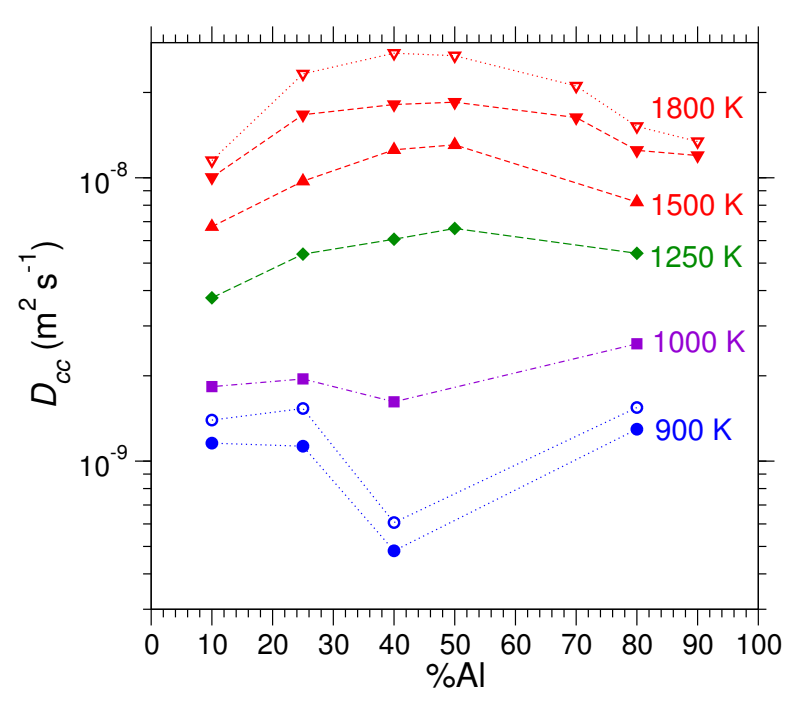

FIG. 1. Interdiffusion coefficient $D_{c c}$ in a model Al-Ni system, from molecular-dynamics computer simulations, as a function of $\mathrm{Al}$ concentration at various fixed temperatures (filled symbols, as labeled). Open symbols show $D_{c c}$ estimated from the Darken relation, Eq. 11) (without a correction, $S=1$ ) for $T=1800 \mathrm{~K}$ and $T=900 \mathrm{~K}$.

of classical liquid-state theory 30148 . It may in fact obey opposite mixing trends as the MCT contribution. In the following, we focus on the low-temperature dynamics, so that this omission will not change the results qualitatively.

\section{RESULTS}

\section{A. Al-Ni Simulations}

Figure 1 shows MD simulation results for the interdiffusion coefficient in $\mathrm{Al}_{x} \mathrm{Ni}_{100-x}$ alloys as a function of the number concentration $x$ of $\mathrm{Al}$ atoms. Different curves correspond to changing concentration at fixed temperature. The data extend those discussed in Ref. 34, where only a single temperature was studied. As the temperature is lowered, interdiffusion becomes slower at any concentration. This is the typical trend for all kinetic transport coefficients, and has been discussed for the present simulation before ${ }^{20}$.

At high temperatures, $T=1500 \mathrm{~K}$, say, one observes a maximum in the interdiffusion coefficient $D_{c c}$ as a function of composition: for $\mathrm{Al}$ concentrations around $x \approx 0.4$ to $x \approx 0.5$, interdiffusion is enhanced by roughly a factor of 2 with respect to the systems containing only a small concentration of either $\mathrm{Al}$ or $\mathrm{Ni}$.

This concentration dependence of $D_{c c}$ changes qualitatively as a function of temperature. Approaching $T \approx 1000 \mathrm{~K}$, the maximum observed at higher temperatures vanishes, and at $T=900 \mathrm{~K}$ instead of a maximum, a minimum is observed at $x \approx 0.4$. At this temperature, interdiffusion is more than a factor 2 slower in the

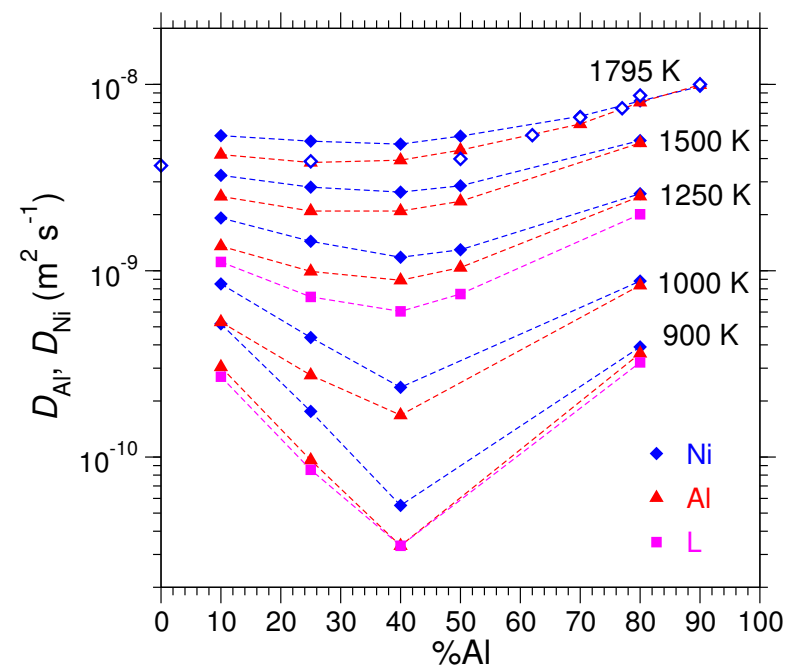

FIG. 2. Al- (filled triangles) and Ni- (filled diamonds) self diffusion coefficients of the model Al-Ni system from MD simulations, as a function of $\mathrm{Al}$ concentration at various temperatures as labeled. For the temperatures $T=1250 \mathrm{~K}$ and $900 \mathrm{~K}$, also the Onsager coefficient $L$ for interdiffusion is shown (squares). Experimental data for $D_{\mathrm{Ni}}^{s}$ at $T=1795 \mathrm{~K}$ obtained from quasielastic neutron scattering (Ref. [35) are shown as open diamonds.

$\mathrm{Al}_{40} \mathrm{Ni}_{60}$ system than in any of the almost pure systems. This change from a maximum in $D_{c c}$ due to mixing, to a minimum due to mixing, is a main subject for the following discussion.

Also shown in Fig. 1 are estimates of $D_{c c}$ according to the Darken equation, Eq. (1) with $S=1$ (open symbols). While the concentration dependence is captured qualitatively correctly, the Darken equation significantly overestimates $D_{c c}$. This effect is more pronounced at higher temperatures, while the quantitative error made by Eq. (1) is less at lower temperatures. To rationalize this is a second main point for the discussion below.

Other than the interdiffusion coefficient, the selfdiffusion coefficients in the MD simulation always display a minimum as a function of concentration at fixed temperature, in the whole range we investigated. This is shown in Fig. 2 As a generic trend, $\mathrm{Ni}$ diffusion is slightly faster, but on the Al-rich side, the difference is much less pronounced. For $T=1795 \mathrm{~K}$, a shallow minimum in both self-diffusion coefficients is seen on the Ni-rich side. Up to $x_{\mathrm{Al}} \approx 0.5$, the $D_{\alpha}^{s}$ depend only weakly on concentration: this is compatible with recent experimental data on Ni-self diffusion obtained from quasielastic neutron scattering ${ }^{35}$ (open diamonds in Fig. 2). The MD simulation systematically overestimates self-diffusion coefficients on the $\mathrm{Ni}$ rich side. For example, for $T=(1514 \pm 5) \mathrm{K}$, a value of $D_{\mathrm{Ni}}^{s} \approx(2.09 \pm 0.08) \mathrm{m}^{2} / \mathrm{s}$ was measured in pure $\mathrm{Ni}$ using quasi-elastic neutron scattering combined with electromagnetic levitation $\frac{49}{4}$; the value estimated from Fig. 2 is about a factor of 3 higher. On the Al-rich side, long- 


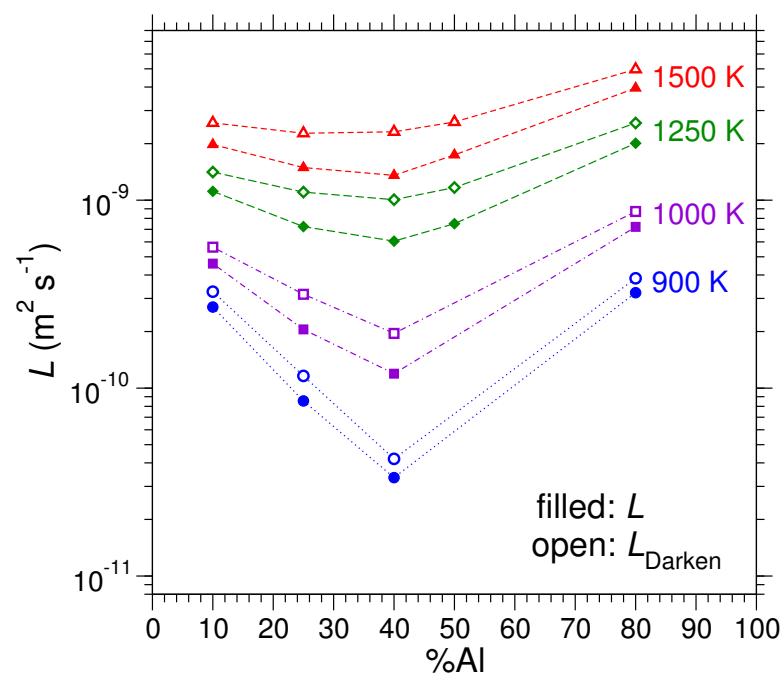

FIG. 3. Onsager kinetic coefficient for interdiffusion, $L$, in the Al-Ni MD computer simulation, as a function of $\mathrm{Al}$ concentration at various fixed temperatures as indicated (filled symbols). Open symbols represent the estimate based on only the self-diffusion coefficients according to the Darken equation.

capillary experiments under microgravity conditions 50 obtained $D_{\mathrm{Ni}}^{s} \approx 3.7 \times 10^{-9} \mathrm{~m}^{2} / \mathrm{s}$ in (almost pure) Al at $T=969 \mathrm{~K}$. This value is (if somewhat high) compatible with our MD results. Note however, that uncertainties, e.g., relating to temperature control may be considerable in this experiment. The MD simulation extends the temperature window covered in the mentioned experiments to considerably lower temperatures. Upon decreasing the temperature, the intermediate-concentration minimum in both self-diffusion coefficients becomes more pronounced and moves towards higher Al-concentrations (about $x_{\mathrm{Al}} \approx 0.4$ ). At $T=900 \mathrm{~K}$, it amounts to about a factor 10 decrease.

The behavior of the self-diffusion coefficients already indicates the mechanism responsible for the change of the concentration-dependence of the interdiffusion coefficient from a maximum due to mixing, to a minimum due to mixing. Recall that $D_{c c}$ is decomposed into a kinetic part, the Onsager coefficient $L$, and a thermodynamic driving force, $\Phi$. In the dense melt, one expects that qualitatively, all kinetic factors behave similarly, because relaxation at high densities and/or low temperatures is a strongly cooperative process. Figure 3 shows the kinetic contribution to $D_{c c}$ as filled symbols. At all temperatures, the Onsager coefficient exhibits a minimum at intermediate concentrations. This minimum becomes more pronounced upon lowering the temperature, similar to the trend already observed for the self-diffusion coefficients. At the lowest temperature investigated here, $T=900 \mathrm{~K}$, the suppression of the interdiffusion kinetics upon mixing is almost a factor of 8 , i.e., even more than the suppression of the interdiffusion coefficient. From this observation, one already infers that the thermodynamic

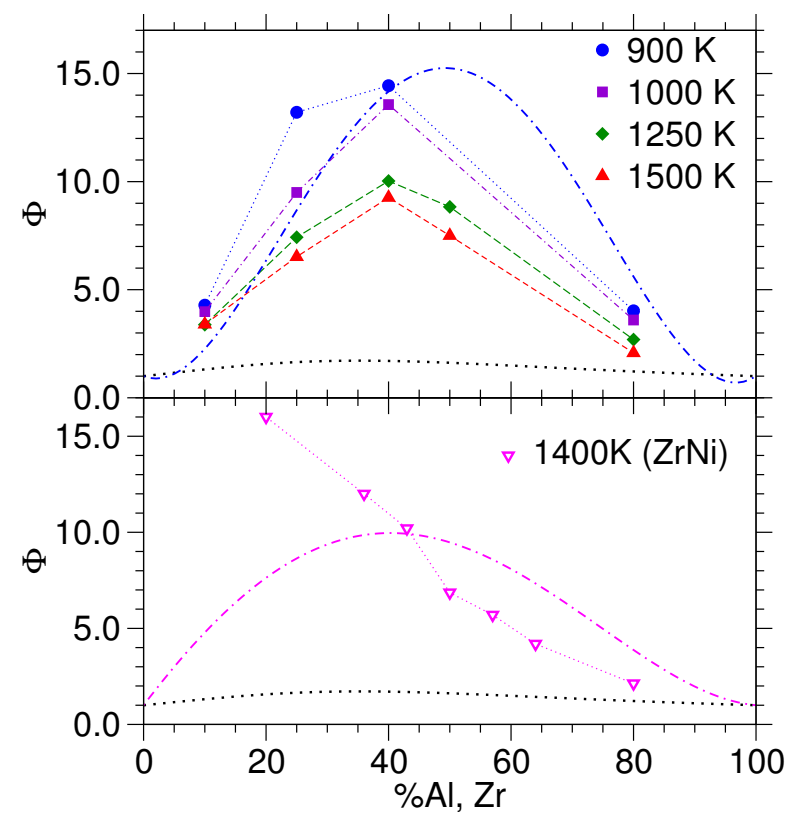

FIG. 4. Thermodynamic factor $\Phi$ connecting the Onsager coefficient $L$ and the interdiffusion coefficient $D_{c c}$. Results from molecular-dynamics simulations for a model Al-Ni system are shown as filled symbols as functions of $\mathrm{Al}$ concentration (upper panel; constant temperatures as indicated). Open symbols (lower panel) are results from MD simulations of a model $\mathrm{Zr}-\mathrm{Ni}$ system, as function of $\mathrm{Zr}$ concentration. Dotted lines indicate the pure entropic hard-sphere contribution expected for strongly different interaction radii, at constant density. Dash-dotted lines are the results of thermodynamic modeling (Refs. 51 and 52) for Al-Ni at $T=900 \mathrm{~K}$ respectively for $\mathrm{Zr}-\mathrm{Ni}$ at $T=1400 \mathrm{~K}$.

factor $\Phi$ must display a maximum as a function of concentration. Again, the minimum in the kinetic coefficient $L$ is observed near the composition with $x \approx 0.4$.

The corresponding concentration dependence of the thermodynamic factor $\Phi$ is shown in Fig. 4. As expected from the above discussion, a maximum is found for all temperatures, again at concentrations near $x=0.4$. For the lowest temperature shown, an almost 15 -fold increase with respect to the pure systems is found. The increase compared to the lowest Al- respective Ni-concentrations investigated in our MD simulations, is roughly a factor of 3. At higher temperatures, a similar maximum in $\Phi$ is seen, albeit less pronounced. The results from our MD simulation agree well with a recent $a b$ initio molecular dynamics study for the $x=0.8$ composition ${ }^{53}$.

A standard empirical method to obtain thermodynamic factors is the so-called CALPHAD method: based on large databases of experimentally determined thermodynamic properties, suitable interpolation techniques are used to reconstruct the Gibbs free energy $G$. For binary systems, interpolation polynomials up to second order in $\left(x_{\mathrm{A}}-x_{\mathrm{B}}\right)$ with linearly $T$-dependent coefficients (so-called Redlich-Kister polynomials) are usually employed. For the Al-Ni liquid, parameters were deter- 
mined by Huang and Chang 51 . The resulting thermodynamic factor $\Phi$ is shown in Fig. 4 as a dash-dotted line (for $T=900 \mathrm{~K}$ ). Agreement with our MD simulations is fair, and the magnitude of the increase in $\Phi$ is captured correctly. The interpolation assumed in Ref. 51 implies that $\Phi(x)$ is almost symmetric around $x \approx 1 / 2$, and has its maximum there. Our MD simulation indicates that the maximum in $\Phi(x)$ is shifted somewhat to the Ni-rich side, as was already noted for a higher temperature earlier ${ }^{34}$. Furthermore for Al-Ni, the CALPHAD calculations suggest $\Phi<1$ if one of the components is very dilute. The investigation of this parameter regime in experiment and simulation is the subject of a separate publication 54 . Note also that the parametrization of Ref. 51 is constructed to work in the stable liquid phase, while we use it to smoothly extrapolate to the metastable supercooled liquid.

The overall temperature variation of the thermodynamic factor at a fixed concentration is weaker than that of the Onsager coefficient. In addition, it is reversed: while kinetic coefficients like $L$ or the self-diffusion coefficients drop sharply with decreasing temperature, the thermodynamic factor increases. Taken together, at high temperatures the maximum in the thermodynamic factor dominates the behavior of $D_{c c}=L \cdot \Phi$, so that a maximum in the interdiffusion coefficient is observed. At low temperatures, the stronger suppression and more pronounced minimum in $L$ dominates, resulting in a minimum in the interdiffusion coefficient although the maximum in the thermodynamic factor becomes more pronounced.

That the thermodynamic factor exhibits a maximum is expected to be a rather generic effect: deviations from ideal-gas-like mixing in a thermodynamically stable system that favors mixing will generate thermodynamic driving forces acting to level out concentration fluctuations. This corresponds to a thermodynamic factor $\Phi>1$. Such a trend is even observed without any attractive interactions and on purely entropic grounds: already the hard-sphere system displays a mixing-induced maximum in $\Phi$. Although more accurate empirical equations of state are available for hard-sphere mixtures, the effect is indicated in the Percus-Yevick (PY) approximation for the static structure factor ${ }^{21}$. Since PY approximates the system to be stable and mixing for all parameters, one can discuss its thermodynamic factor for simplicity in the limiting case of vanishing size of one of the species. Evaluating $\Phi=x(1-x) / S_{c c}(q=0)$ with $x$ indicating the large-particle number concentration, one gets for size ratio $\delta \ll 1$

$$
\Phi_{\mathrm{HSPY}, \delta \rightarrow 0}=\frac{\left(1+\frac{\pi}{3} x \rho\right)^{2}}{1+\frac{\pi}{6} x \rho\left(6 x+\frac{\pi}{6} x \rho(1+3 x)-2\right)} .
$$

This result is shown as a dotted line in Fig. 4. It exhibits a maximum value of approximately 1.7 at a concentration $x \approx 0.35$. The observed thermodynamic factors in metallic melts are typically much larger, which may be intuitively explained by the lack of inter-species attractions

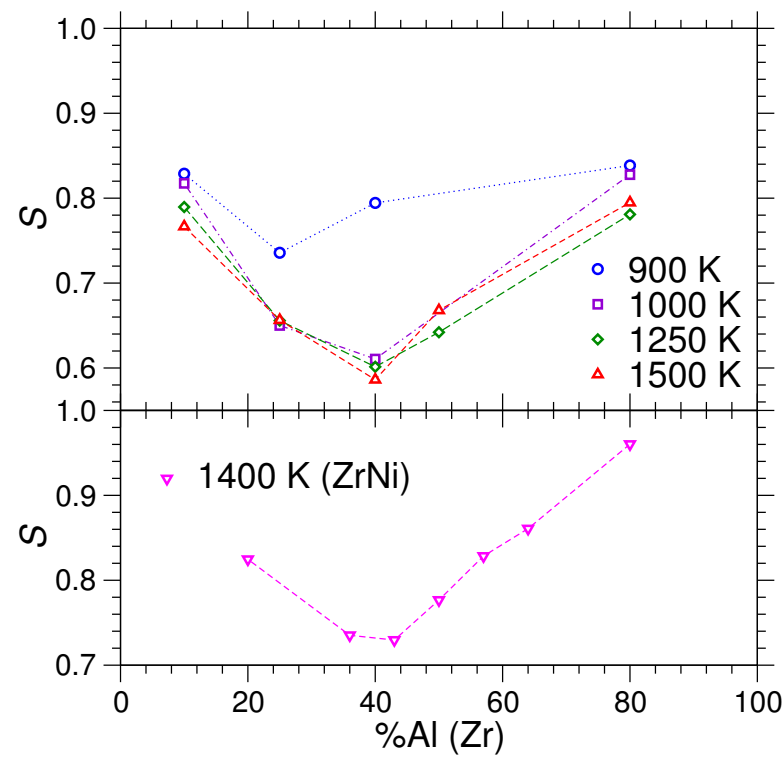

FIG. 5. Correction factor $S$ for the Darken equation obtained from MD computer simulation of Al-Ni and Zr-Ni models, as a function of $\mathrm{Al}$ respective $\mathrm{Zr}$ concentration, for the temperatures indicated.

and non-additive mixing leading to a much suppressed chemical ordering in hard-sphere mixtures ${ }^{32}$. It is nevertheless remarkable that the maximum position for $\Phi$ at concentrations in the range around $40 \%$ of the larger particles may be rather generic whenever the system is dense enough so that the core-repulsion contribution from the interparticle interactions becomes significant.

As will be discussed below in connection with MCT, a strong decrease in the kinetic coefficients due to mixing is also expected to be rather generic in dense systems, as it also arises already in the hard-sphere mixture model. The evolution of the concentration dependence in the interdiffusion coefficient highlighted in Fig. 1 should therefore be typical for dense metallic melts, given that the relevant temperature and concentration ranges can be explored without intervening phase separation, crystallization, or other effects that prevent the (supercooled) liquid state to be accessed.

By equating the Onsager coefficient of interdiffusion to the weighted sum of the self-diffusion contributions, the Darken equation expresses the assumption that all kinetic contributions to diffusion processes share the same physical mechanism. In the MD simulation, Darken's assumption is easily tested. Figure 3 includes as open symbols the Darken average. It is seen that the Darken equation in $\mathrm{Al}-\mathrm{Ni}$ systematically overestimates the Onsager coefficient. This is also evident already from Fig. 2, where the Onsager coefficient $L$ is shown for two exemplary temperatures. It is smaller than either of the self-diffusion coefficients, in contradiction to Darken's Eq. (1) with $S=1$. The qualitative trend of the Onsager coefficient both with changing temperature and changing concentration is captured by the Darken approxima- 


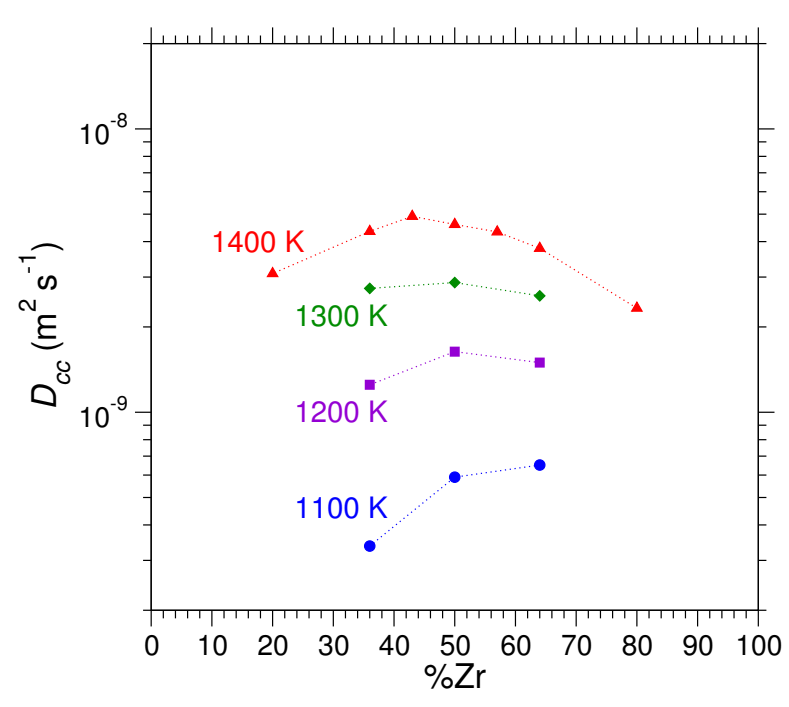

FIG. 6. Interdiffusion coefficients $D_{c c}$ of a $\mathrm{Zr}-\mathrm{Ni}$ model system from MD computer simulation, at various fixed temperatures as indicated and as a function of $\mathrm{Zr}$-concentration.

tion. This is a consequence of the fact that all kinetic, non-thermodynamic, contributions to the diffusion coefficients slow down with decreasing temperature and with concentration approaching $x \approx 40 \%$.

The quality of the Darken approximation is best visualized in terms of the correction factor $S=L /\left(x_{\mathrm{B}} D_{\mathrm{A}}^{s}+\right.$ $\left.x_{\mathrm{A}} D_{\mathrm{B}}^{s}\right)$. This is shown in Fig. 5. As already evident from Fig. 3, $S \leq 1$ holds for all compositions and temperatures investigated in our Al-Ni simulations. At high temperatures and intermediate concentrations, $S \approx 0.6$, i.e., the Darken approximation overestimates the interdiffusion coefficient by about $66 \%$. By construction, the agreement becomes better if either $x \approx 0$ or $x \approx 1$ is approached. There, $S=1$ should hold on theoretical grounds. At the lowest temperature, $T=900 \mathrm{~K}$, the correction factor is significantly closer to unity at all concentrations, obeying $S \approx 0.8$ (corresponding to a $25 \%$ over-estimation by Darken's approximation). This is in line with the expectation that the lower the temperature, the stronger the cooperative relaxation effects that imply a tight coupling between self- and collective diffusion processes. As explained below, this is the picture emerging from mode-coupling theory as the temperature is lowered towards the kinetic glass transition. According to our MD simulatons, there is a marked increase in $S$ between $T=1000 \mathrm{~K}$ and $T=900 \mathrm{~K}$. Note that coincidentially, the concentration dependence of the $D_{c c}$ starts to be dominated by kinetic effects around $T=900 \mathrm{~K}$ (it displays a minimum as a function of concentration as seen in Fig. 1), while it is still dominated by thermodynamic effects at $T=1000 \mathrm{~K}$.

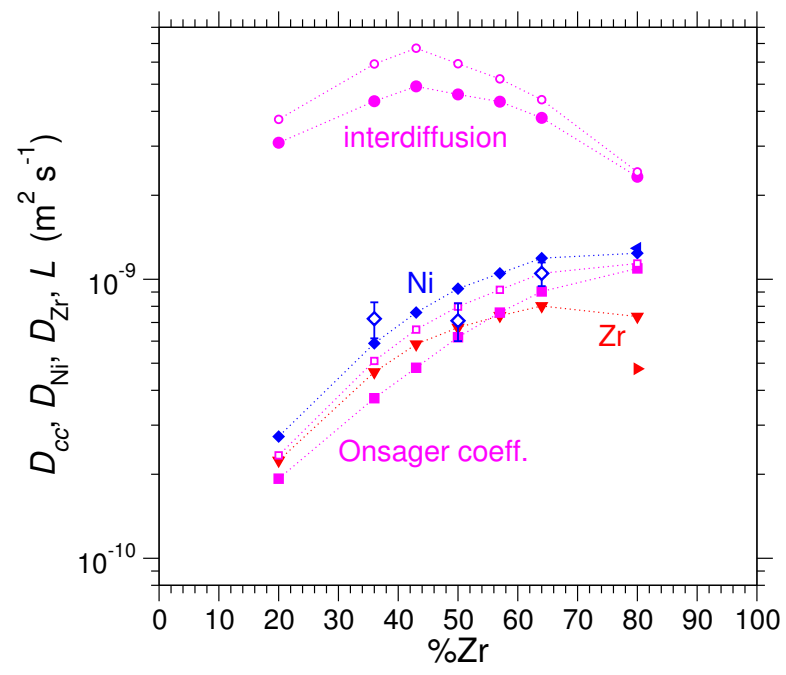

FIG. 7. Interdiffusion coefficient $D_{c c}$ (filled circles), related Onsager kinetic coefficient $L$ (filled squares), and self-diffusion coefficients for $\mathrm{Ni}$ and $\mathrm{Zr}$ (filled diamonds and inverted triangles), for the $\mathrm{Zr}-\mathrm{Ni}$ simulation model at fixed temperature $T=1400 \mathrm{~K}$, as a function of $\mathrm{Zr}$ concentration. Open squares and circles are the Onsager coefficient and $D_{c c}$ estimated from the Darken equation. Left- and right-pointing triangles are the self-diffusion coefficients for $\mathrm{Ni}$ and $\mathrm{Zr}$, obtained using a different MD simulation model from Ref. 42 Open diamonds with error bars are experimental data for $D_{\mathrm{Ni}}$ from quasielastic neutron scattering (interpolated from Ref. [55).

\section{B. Zr-Ni Simulations}

To put our findings for the Al-Ni model in context, we next discuss results from molecular-dynamics simulations on a $\mathrm{Zr}-\mathrm{Ni}$ model system. As mentioned above, these two binary mixtures display quite different thermodynamic phase diagrams, and are representatives for mixtures with different effective-size ratios in terms of their kinetics. However, the qualitative arguments given above in the case of $\mathrm{Al}-\mathrm{Ni}$, are expected to be generic, so that a test for $\mathrm{Zr}-\mathrm{Ni}$ as a second representative example is in order.

Figure 6 shows results for the interdiffusion coefficient $D_{c c}$ to be compared to the corresponding $\mathrm{Al}-\mathrm{Ni}$ result in Fig. 1. Keeping in mind that for the $\mathrm{Zr}-\mathrm{Ni}$ model, MD simulations were only carried out for $x \geq 0.36$ at low temperatures, one indeed observes qualitative similarities between the two systems. For high temperatures, $D_{c c}$ in the $\mathrm{Zr}-\mathrm{Ni}$ model decreases displays a maximum around $x \approx 0.4$. At the lower temperatures, the MD data do not allow to draw a definite conclusion, but they are compatible with the reduction of this maximum and the development of a minimum at some $x \lesssim 0.4$.

In Fig. 7, we show all diffusion coefficients, $D_{c c}, D_{\mathrm{Ni}}^{s}$, and $D_{\mathrm{Zr}}^{s}$, evaluated from the $\mathrm{Zr}-\mathrm{Ni}$ MD simulation model at the fixed temperature $T=1400 \mathrm{~K}$. In difference to the $\mathrm{Al}-\mathrm{Ni}$ simulation discussed above, the self-diffusion coefficients show a notable increase with increasing $\mathrm{Zr}$ 
concentration. Experimental values for $D_{\mathrm{Ni}}^{s}$ have been obtained by quasielastic neutron scattering and compiled in Ref. 55. Agreement with the MD simulation is reasonable, but not surprising since the simulation potential has been tuned based on the diffusion data in $\mathrm{Zr}_{64} \mathrm{Ni}_{36}$. Experimental values for $\mathrm{Zr}_{50} \mathrm{Ni}_{50}$ are noticeably lower than expected from the simulation; the reason for this discrepancy is unclear. For $x_{\mathrm{Zr}}=0.36$, the experimental value is above the one calculated from the simulation. Recall that for pure $\mathrm{Ni}$, experiments ${ }^{49}$ suggest a value $D_{\mathrm{Ni}}^{s} \gtrsim 1 \times 10^{-9} \mathrm{~m}^{2} / \mathrm{s}$, significantly higher than the MDsimulated value for the lowest $\mathrm{Zr}$-concentration that is accessible without crystallization. This may suggest that the $\mathrm{Zr}$-Ni model employed in our MD simulations underestimates the diffusivities on the Ni-rich side. On the other hand, the model suggests strong concentration dependence of some quantities in the range $x_{\mathrm{Zr}} \leq 0.2$, as will be discussed below in connection with the thermodynamic factor shown in Fig. 4. In this case, a pronounced minimum in $D_{\alpha}^{s}$ would occur in this concentration regime.

At all concentrations, one has $D_{\mathrm{Zr}}^{s}<D_{\mathrm{Ni}}^{s}$, which is expected from the simple analogy to hard-sphere mixtures since Ni is the "smaller" atom. Previous calculations using MCT based on static structure factors determined by neutron scattering ${ }^{\sqrt{32}}$ suggested that $D_{\mathrm{Zr}}^{s} \approx D_{\mathrm{Ni}}^{s}$ holds at least for $T=1350 \mathrm{~K}$ and $x_{\mathrm{Zr}}=0.64$. These static structure factors showed a strong pre-peak indicating strong chemical short-range order that suppresses the decoupling of $\mathrm{Ni}$ and $\mathrm{Zr}$ diffusion one expects from the purely entropic hard-sphere mixture. In our MD simulation model, this pre-peak is much less pronounced. The enhancement of $D_{\mathrm{Ni}}^{s}$ over $D_{\mathrm{Zr}}^{s}$ in the simulation increases with increasing $\mathrm{Zr}$ concentration, up to $D_{\mathrm{Ni}}^{s} \approx 1.7 D_{\mathrm{Zr}}^{s}$ at $x_{\mathrm{Zr}}=0.8$. This compares reasonably well with the factor 2.5 reported for a different $\mathrm{Zr}-\mathrm{Ni}$ model by Mutiara and Teichler $\stackrel{42}{ }$. Values from this study are shown in Fig. 7 as left- and right-pointing triangle symbols.

Again, for the Zr-Ni system the MD simulation allows to separate the thermodynamic factor $\Phi$ and the correction factor $S$ from the overall interdiffusion coefficient. Concentration dependent results at fixed temperature from our Zr-Ni simulations are shown together with the Al-Ni results in Figs. 4 and 5. One confirms that both terms are of equal magnitude in the two systems. Again, the correction factor $S$ displays a minimum for concentrations corresponding to roughly $60 \% \mathrm{Ni}$. At the same time, the values for $S$ obtained in the $\mathrm{Zr}-\mathrm{Ni}$ simulation are somewhat higher than in Al-Ni at the same temperature, approaching unity on the Zr-rich side for $T=1400 \mathrm{~K}$. Here one has to keep in mind that at equal temperatures, the $\mathrm{Zr}-\mathrm{Ni}$ system is kinetically slower, as evidenced from Figs. 2 and 7. Assuming that slow cooperative relaxation processes are responsible for a strong coupling between all modes of diffusion, and hence drive $S$ towards unity, this rationalizes the better quantitative (and similar qualitative) performance of Darken's approximation in the $\mathrm{Zr}-\mathrm{Ni}$ melt.

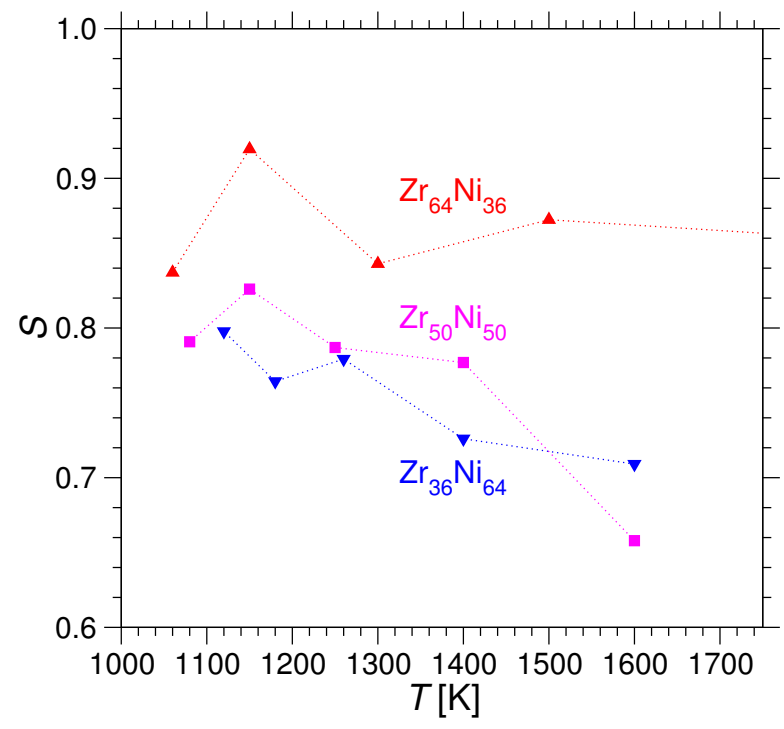

FIG. 8. Correction factor $S$ for the Darken equation for the interdiffusion coefficient in the Zr-Ni MD simulation model for three different compositions, as a function of temperature.

The thermodynamic factor in our $\mathrm{Zr}-\mathrm{Ni}$ model at $T=1400 \mathrm{~K}$ monotonically increases over the concentration range covered in the simulations $(x \geq 0.2)$. Since $\Phi \rightarrow 1$ should hold for $x \rightarrow 0$, we conclude that the thermodynamic factor displays a pronounced maximum in the Zr-concentration interval $x_{\mathrm{Zr}} \in[0,0.2]$. Rapid crystallization in the simulation prevents us from checking this directly. Such a maximum is at significantly lower concentration than the maximum in $\Phi$ found in the Al-Ni system. The estimate based on interpolation of thermodynamic property data, with parameters for Redlich-Kister polynomials obtained by Ghosh ${ }^{52}$ (shown in Fig. 4 as a dash-dotted line), predicts the maximum to be shifted to the Ni-rich side compared to Al-Ni, albeit not as much as suggested by the MD simulation. The reason for the strong increase observed in the simulation is not clear. We could not find obvious structural changes in the melt compared to other concentrations.

For the Zr-Ni system, we also show the temperature dependence of the correction factor $S$ and the thermodynamic factor $\Phi$, in Fig. 8 respectively 9, at the three compositions $x=0.36, x=0.5$, and $x=0.64$ for which a large temperature window was studied in our simulations. Turning first to the dynamic correction factor $S$, we find a week increase with decreasing temperature indicating the crossover from typical liquid-state dynamics at high temperatures to a cage-effect dominated regime (where one expects $S$ to approach unity). However, it has to be noted that the temperature variation of $S$ is rather weak, and in particular for the $\mathrm{Zr}_{64} \mathrm{Ni}_{36}$ system, no obvious trend can be seen within the error bars of our simulation. This is similar to what has been observed in $\mathrm{Al}_{80} \mathrm{Ni}_{20}$ over a similar temperature range $\mathrm{e}^{20}$.

The thermodynamic factor, Fig. 9, increases with decreasing temperature as naively expected. This increase 


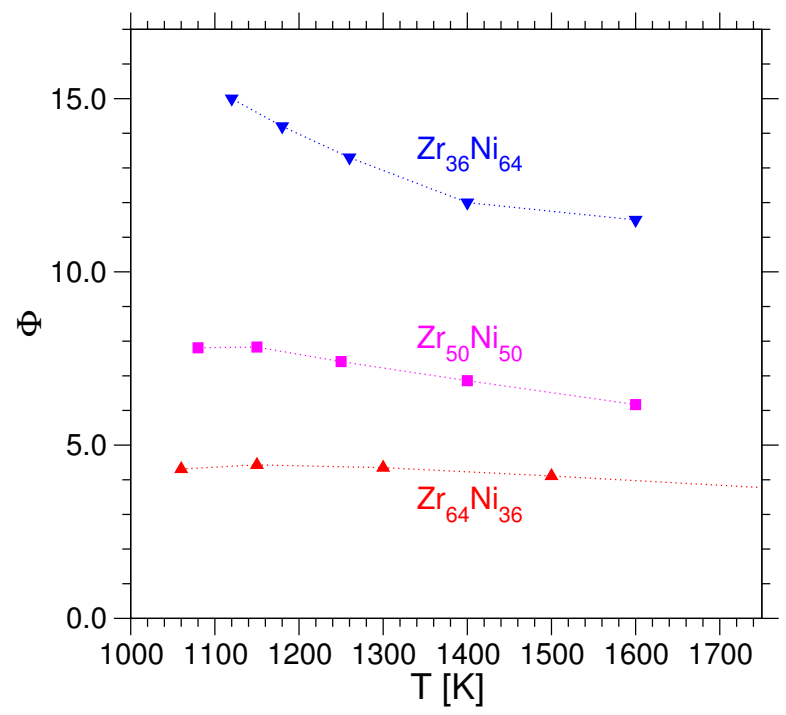

FIG. 9. Thermodynamic factor $\Phi$ for interdiffusion in the $\mathrm{Zr-Ni}$ model for three different compositions as labeled, as a function of temperature.

is more pronounced for the $\mathrm{Zr}_{36} \mathrm{Ni}_{64}$ system, i.e., closer to the composition where a maximum in $\Phi$ is expected as a function of composition. Overall, the values of $\Phi$ extracted from our simulation are compatible with those estimated from experiment ${ }^{32}$. They are somewhat lower than the large thermodynamic factor reported for the amorphous $\mathrm{Zr}_{43} \mathrm{Ni}_{57}$ solid 56 , which is however to be expected since we are here dealing with the liquid state at much larger temperatures.

\section{MCT Results}

The development of a pronounced minimum in the kinetic transport coefficients at intermediate concentrations and low enough temperatures can be rationalized within the mode-coupling theory of the glass transition. It has already been noted that for hard-sphere mixtures of not too disparate size, MCT predicts a favorization of the glass by mixing 29 . The total packing fraction at the kinetic glass transition in this regime is decreased by mixing, such that along a cut of constant packing fraction, increasing the minority-species concentration towards roughly $x \approx 1 / 2$ will decrease the controlparameter distance to the glass transition. This translates into a suppression of kinetic transport coefficients as a function of species concentration at fixed packing fraction, as confirmed in event-driven MD simulations of binary hard-sphere mixtures 30 . This suppression holds for size ratios $0.75 \lesssim \delta<1$ and arises from the increased structural disorder introduced by the minority species into the pure system. It is remarkable since for colloidal suspensions, where mixtures with stronger size disparity are typical, the opposite effect is usually discussed and rationalized by effective interactions induced

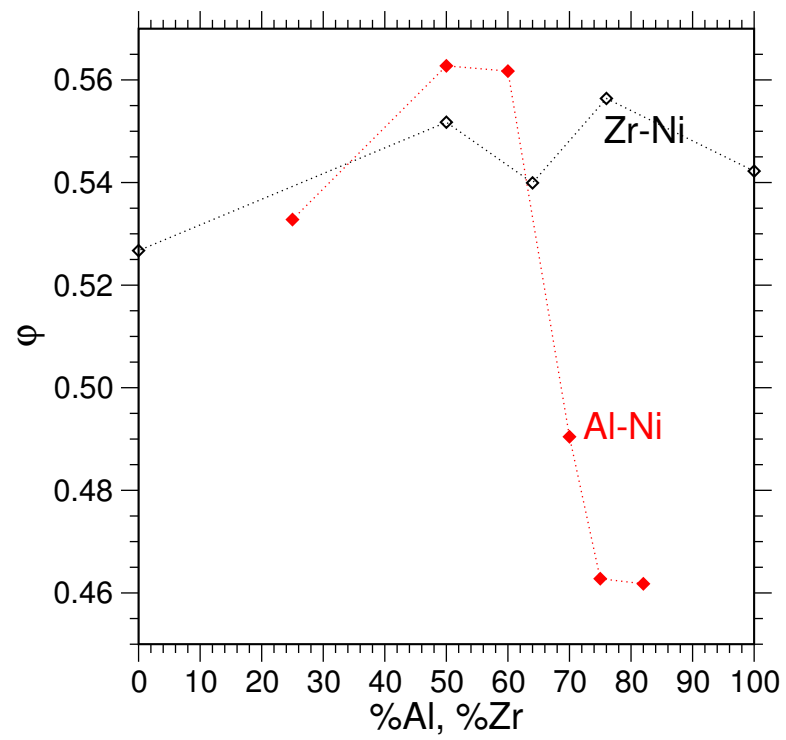

FIG. 10. Effective packing fractions $\varphi$ estimated for various Al-Ni (filled symbols) and $\mathrm{Zr}-\mathrm{Ni}$ (open symbols) melts, at $T=1473 \mathrm{~K}$ for various compositions.

entropically between the large-species particles ${ }^{47}$. In this case, the kinetic coefficients typically show a maximum at intermediate concentrations and fixed volume fraction, equivalent to a strong fluidization of the system by mixing. However, at size ratios close to unity, the depletioninteraction effect is no longer the dominant mechanism, since here the minority species makes a strong contribution to the static structure that is not accounted for in the depletion picture.

A rough estimate of entropic size effects in metallic melts is provided by the covalent radii of the species. Estimated values 57 are $R_{\mathrm{Zr}}=1.45 \AA, R_{\mathrm{Ni}}=1.15 \AA$, and $R_{\mathrm{Al}}=1.25 \AA$. From this, size ratios are evaluated (including error bars obtained from different estimates of the radii based on crystallography $\sqrt{58}, R_{\mathrm{Zr}}=1.75 \AA$, $R_{\mathrm{Ni}}=1.24 \AA$, and $\left.R_{\mathrm{Al}}=1.21 \AA\right): \delta_{\mathrm{Al}-\mathrm{Ni}} \approx 0.92 \pm 0.06$, and $\delta_{\mathrm{Zr}-\mathrm{Ni}} \approx 0.79 \pm 0.08$. Based on this estimate, the kinetic slowing down upon mixing is indeed expected in both systems upon entropic grounds, since $\delta \gtrsim 0.75$ (as is the case for metallic melts quite generally).

Assuming the constituents of the dense melt to be reasonably close to hard spheres, one can assign to it an effective packing fraction.5. Based on the above diameters and the relative atomic masses, $m_{\mathrm{Zr}}=91.224 \mathrm{amu}$, $m_{\mathrm{Ni}}=58.693 \mathrm{amu}$, and $m_{\mathrm{Al}}=26.982 \mathrm{amu}$, the packing fraction $\varphi$ can be estimated from existing experimental data for the mass density. The results for Al-Ni and $\mathrm{Zr}-\mathrm{Ni}$ are shown in Fig. 10. Here, mass-density data have been taken from the literature ${ }^{59 / 60}$ and (in the case of $\mathrm{Zr}-\mathrm{Ni}$ ) extrapolated to constant temperature. For the case of $\mathrm{Al}-\mathrm{Ni}$, one notices a marked maximum around $x_{\mathrm{Al}} \approx 0.5$. This agrees qualitatively with the above result, that the dynamics is slowest around this composition: for the estimated size ratio, the hard-sphere packing fraction corre- 


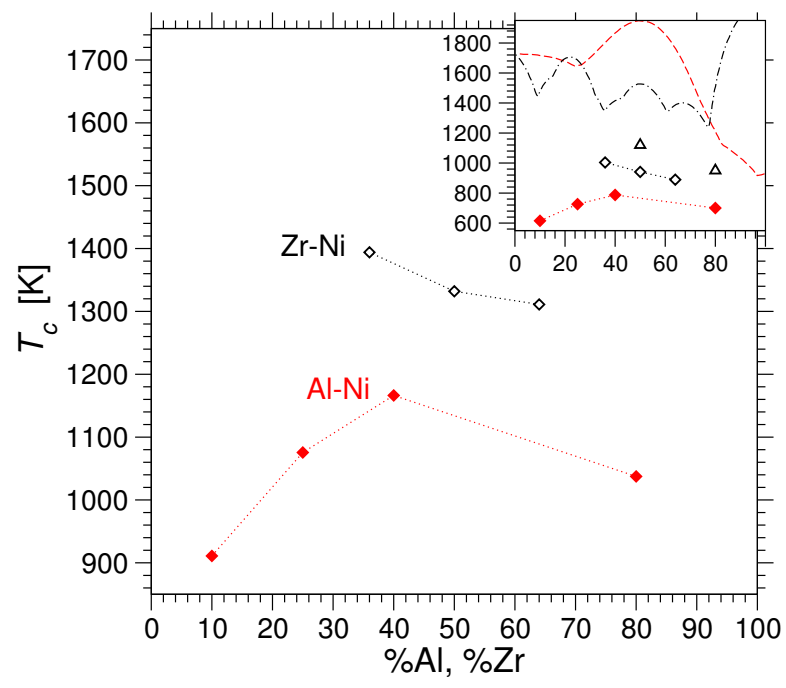

FIG. 11. Kinetic glass transition temperature $T_{c}$ for Al$\mathrm{Ni}$ (filled symbolds) and $\mathrm{Zr}-\mathrm{Ni}$ (open symbols) as a function of composition, obtained from mode-coupling theory of the glass transition (MCT) using the static structure factors from $\mathrm{MD}$ simulation. The inset shows the estimates of $T_{c}^{\mathrm{MD}}$ from the MD simulation directly (see text for details), together with boundaries for the thermodynamic-equilibrium liquid phase (Al-Ni: dashed; Zr-Ni: dash-dotted). Triangles mark the $T_{c}^{\mathrm{MD}}$ values obtained from a different $\mathrm{Zr}-\mathrm{Ni}$ potential in Refs. 41 and 42 .

sponding to the MCT glass transition slightly decreases, while at the same time the effective packing fraction at constant temperature increases. Both these effects suggest that, entropically, the system is closer to arrest for $x \approx 0.5$ than for other compositions. For $\mathrm{Zr}-\mathrm{Ni}$, the data do not allow to identify a clear trend. However, the effective packing fractions in $\mathrm{Zr}-\mathrm{Ni}$ are larger than those of $\mathrm{Al}-\mathrm{Ni}$ especially on the $\mathrm{Ni}$-poor side; this agrees with our finding that the dynamics in $\mathrm{Zr}-\mathrm{Ni}$ is slower than that in $\mathrm{Al}-\mathrm{Ni}$ at the same temperature.

Without resorting to the hard-sphere analogy, one can for the MD-simulated metallic melts directly test the change of the MCT glass-transition temperature $T_{c}$ as a function of composition. This is shown in Fig. 11 for the Al-Ni model system. $T_{c}(x)$ exhibits a maximum at intermediate $x$, so that along an isotherm above $T_{c}$, the distance to the glass transition is decreased upon approaching these intermediate- $x$ compositions. This is in analogy to the discussion of the hard-sphere mixtures above, and thus appears to be driven by mainly entropic effects.

We also show in Fig. 11 the values obtained from a MCT calculation using the $\mathrm{Zr}-\mathrm{Ni}$ static structure factor from our MD simulation. In line with the finding that the dynamics of $\mathrm{Zr}-\mathrm{Ni}$ is slower than that of $\mathrm{Al}-\mathrm{Ni}$ at the same temperature, the $T_{c}$ values for $\mathrm{Zr}-\mathrm{Ni}$ are systematically higher. Since $x=0$ in both simulations corresponds to a pure Ni system, the corresponding $T_{c}(0)$ values should agree. In light of this, the $T_{c}(x)$ values for $\mathrm{Zr}-\mathrm{Ni}$ will also display a maximum for some $x \leq 0.36$.
The $T_{c}$ values predicted by MCT are not accurate, and typically too high since the theory overestimates the tendency to vitrify. This is a known issue in comparing MCT calculations with MD simulation data ${ }^{45}$. From the MD simulation, $T_{c}$ can be independently estimated by analyzing the intermediate scattering functions in terms of the asymptotic scaling laws provided by $\mathrm{MCT}^{4}$. For the $\mathrm{Zr}-\mathrm{Ni}$ model employed here, the resulting estimates $T_{\mathrm{c}}^{\mathrm{MD}}$ are roughly a factor 1.4 lower than the calculated $T_{c}^{\mathrm{MCT}}$. A similar difference has been observed earlier for the Al$\mathrm{Ni}$ mode 38 . The $T_{c}^{\mathrm{MD}}$ for the two systems are shown in the inset of Fig. 11. There, values obtained using a different Zr-Ni model by Teichler and coworkers ${ }^{41 / 42}$ are added. These values are compatible with the same mixing trend, but are systematically higher. The latter effect is due to our adaption of the EAM potential to match experimentally measured diffusivities. Let us stress that the MCT glass-transition temperature $T_{c}$, being purely kinetic in origin, does not mirror any equilibrium phase transition. In the inset of Fig. 11, dashed (dash-dotted) lines indicate the boundaries of the thermodynamically stable liquid phase for $\mathrm{Al}-\mathrm{Ni}(\mathrm{Zr}-\mathrm{Ni})$, obtained from a standard database ${ }^{61}$. One readily observes that the concentration dependence of $T_{c}$ is not correlated with that of thermodynamic solid-liquid boundary.

Let us add a further remark concerning the idealized $\mathrm{MCT}$ glass transition. According to MCT, the diffusion coefficients approach zero as $T$ approaches $T_{c}$ from above, and the viscosity diverges. This is the case for the ideal glass, which is not attained in real-world experiment, due to residual relaxation processes that are not captured in the MCT approximation. Thus, transport coefficients remain finite even at $T_{c}$ and below. Nevertheless, a strong non-Arrhenius decrease of kinetic coefficients is typically observed in glass formers as one approaches $T_{c}$ from high temperatures. In this sense, the MCT predictions discussed here are not quantitatively accurate, but serve to explain the qualitative features of the slow dynamics in dense metallic melts.

To verify the MCT description, we show in Fig. 12 the diffusion and Onsager coefficients of $\mathrm{Al}-\mathrm{Ni}$ as a function of composition, for fixed temperature. For the MCT calculations, $T=1500 \mathrm{~K}$ and $T=1200 \mathrm{~K}$ were chosen, still above $T_{c}$ at any composition. However, while for $T=1500 \mathrm{~K}$ at $x \rightarrow 0$, the distance to the transition is $\varepsilon=\left(T_{c}-T\right) / T_{c} \approx-0.67$ (using the standard convention that states on the liquid side of the glass transition corresdond to negative distance parameter $\varepsilon$ ), this distance reduces to about $\varepsilon \approx-0.25$ around $x \approx 0.4$. As a result, the self-diffusion and Onsager coefficients decrease by about a factor of 2 between these two compositions. This explains the minimum also seen in all kinetic coefficients of the $\mathrm{Al}-\mathrm{Ni}$ and $\mathrm{Zr}-\mathrm{Ni}$ simulations discussed in conjunction with Figs. 2 and 7 Lowering $T$, as exemplified by the $T=1200 \mathrm{~K}$ result, even smaller absolute values of the distance parameter $\varepsilon$ are reached around $x \approx 0.4$, resulting in a stronger drop of the transport coefficients. 


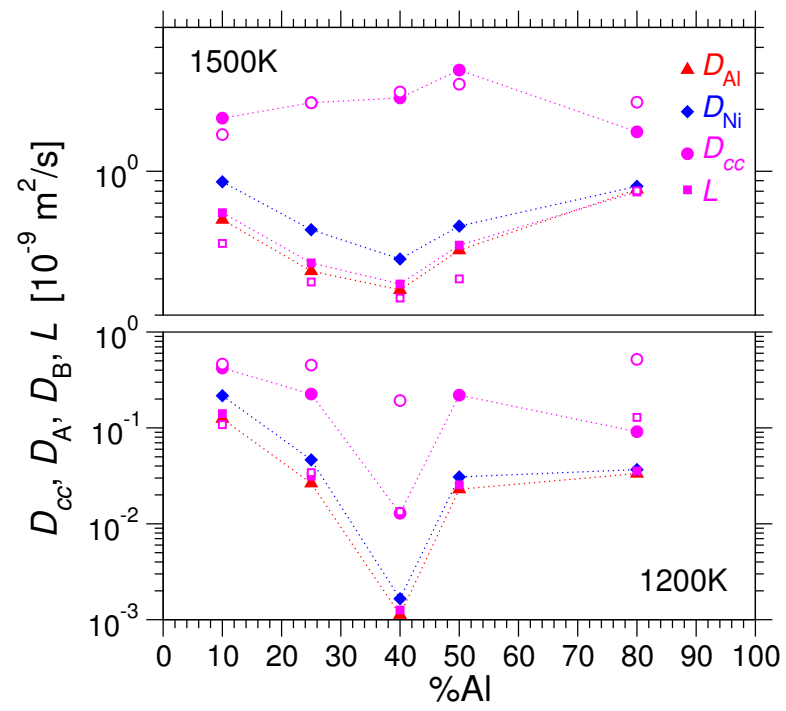

FIG. 12. Self-diffusion coefficients $D_{\alpha}, \alpha=\mathrm{Al}$, Ni, interdiffusion coefficient $D_{c c}$, and the corresponding Onsager coefficient $L$ as functions of Al-concentration, calculated within MCT using the Al-Ni static structure factors from the simulation, at $T=1500 \mathrm{~K}$ (upper panel) and $T=1200 \mathrm{~K}$ (lower panel). MD-simulation results for the interdiffusion coefficient $D_{c c}$ and the corresponding Onsager coefficient $L$ are included as open symbols for $T=1250 \mathrm{~K}$ and $T=900 \mathrm{~K}$, scaled by a factor 0.4 .

The MCT estimate for the interdiffusion coefficient is obtained from the Onsager coefficient multiplied with the thermodynamic factor. The latter is taken directly from the MD-simulated structure factor, and thus is not by itself an MCT prediction. The result for $D_{c c}$ is shown as the filled circles in Fig. 12. Since the suppression of the Onsager coefficient $L$ at $T=1500 \mathrm{~K}$ is about a factor of 2 , but the increase in the thermodynamic factor $\Phi$ amounts to a factor of 3 over the concentration range covered here, cf. Fig. 4, an increase of about a factor 1.5 is observed for $D_{c c}$ over the same concentration range.

Taking this into account, the qualitative agreement of the MCT results with those obtained by the MD simulations discussed above, is quite good. A source of quantitative error is the over-estimation of $T_{c}$ by MCT, as discussed above. It is therefore usual to compare MCT results with those of the MD simulation at a lower temperature. This is not quite accurate for the present discussion, since this overemphasizes the variation in the thermodynamic factor. We therefore have to anticipate that the translation between $T_{\mathrm{MD}}$ and $T_{\mathrm{MCT}}$ where best agreement between simulation and theory is observed, will not be a simple linear relationship. To nevertheless demonstrate the level of qualitative agreement, we include in Fig. $12 \mathrm{MD}$ simulation results for some $T_{\mathrm{MD}}<T_{\mathrm{MCT}}$, to adjust for the discrepancy in $T_{c}$, and additionaly scaled by empirical factors to account for the mismatch in thermodynamic factors at the temperatures used in the comparison. In particular for the higher temperature, $T_{\mathrm{MCT}}=1500 \mathrm{~K}$, the agreement is quite good on a qualitative level.

For the lower temperature $T=1200 \mathrm{~K}$, in MCT both the self-diffusion and Onsager coefficients drop by around two orders of magnitude upon changing the composition. Even the five-fold increase in the thermodynamic factor can no longer compensate for this, so that the interdiffusion coefficient $D_{c c}$ now displays a pronounced minimum around $x \approx 0.4$. Comparing with the MD simulation results for $T_{\mathrm{MD}}=900 \mathrm{~K}$ shown in Fig. 1, and added as rescaled quantities in Fig. 12, this minimum is less strong and less narrow in the simulation. This is expected, since in the region around $x \approx 0.4$, our MCT calculations probe a temperature quite close to $T_{c}$. Here, the additional low-temperature transport processes that MCT neglects, are dominant.

For the MCT results, one even notices a nonmonotonic trend involving a minimum and a subsequent maximum upon increasing $x$ in $D_{c c}$ at $T=1200 \mathrm{~K}$. This is not directly confirmed by MD simulations, but highlights what may happen generically for low temperatures. The concentration of the minimum in the Onsager coefficient $L$ and the concentration of the maximum in the thermodynamic factor $\Phi$ do not necessarily coincide, since they are determined by different physical mechanisms (one kinetic, the other thermodynamic in origin). $D_{c c}$ as the product of these two counter-balancing terms may hence even display multiple minima and maxima.

MCT predicts the Darken approximation to hold much better than what is observed in the simulation. From the theory, for the state points shown in Fig. $12 .|S-1| \lesssim 0.1$ holds. In a sense, the MCT approximation of representing all relevant correlation functions in terms of their overlap with density-relaxation modes overemphasizes the coupling of different dynamical transport mechanisms. Note also that MCT is constructed to describe the slowing down of transport processes as $T_{c}$ is approached. To this end, the theory is centered around an approximation of the growing dynamical friction expressed through its memory kernel. In schematic terms, MCT describes the growth of the friction coefficient for particle motion, $\zeta \rightarrow \infty$, and hence the suppression of diffusivity, $D \sim k T / \zeta \rightarrow 0$. In this sense, MCT is a "slowmode approximation". The Darken equation, Eq. (1), on the other hand is a typical "fast-mode approximation". This conceptual difference between Darken's approach and the mode-coupling theory can lead to very different estimates of the interdiffusion coefficient in particular when the species of the mixture are governed by dynamical processes on very different time scales (such as in mixtures of very disparate species). For the binary metallic melts discussed here, this difference is however not important.

\section{CONCLUSIONS}

We have studied the concentration dependence of selfdiffusion, $D_{\alpha}^{s}$, and interdiffusion, $D_{c c}$, in two dense metal- 
lic melts, Zr-Ni and Al-Ni, by molecular-dynamics simulation and the mode-coupling theory of the glass transition. The two systems were chosen as representatives of metallic melts with very different thermodynamic phase diagrams, involving different eutectica, and rather different glass-forming ability $\underline{62}$. Nevertheless, two generic mixing effects emerge for the mass transport in the (supercooled) liquid state of these non-demixing systems: self diffusion becomes slower upon mixing (i.e., upon increasing the concentration of the minority species towards $x \approx 0.5$ ) while thermodynamic driving forces become stronger upon mixing. The suppression of diffusion kinetics is more pronounced for lower temperatures, in qualitative agreement with MCT.

Interdiffusion is a combination of a kinetic process, quantified by Onsager's coefficient $L$, and a thermodynamic driving force $\Phi$. Consequently, it displays a more subtle mixing scenario. At high temperatures, the enhancement in thermodynamic driving force dominates, leading to a maximum in $D_{c c}$ as a function of concentration. At lower temperatures, the kinetic slowing down is more dominant, causing a minimum in $D_{c c}$. This arises although thermodynamic driving forces increase with decreasing temperature: across the MCT glass transition, thermodynamic quantities such as $\Phi$ change smoothly, while kinetic coefficients exhibit pre-cursors of the idealglass singularity $(1 / L \rightarrow \infty$ in this case). Hence, close to the MCT- $T_{c}$, the kinetic contributions are dominant.

Since thermodynamic and dynamic contributions to interdiffusion exhibit maxima respectively minima at slightly different compositions, the concentration dependence of $D_{c c}$ could in principle be more complicated and lead to $D_{c c}$-versus- $x$ curves with more than one extremum. Indications for this are seen in the MD simulation at low temperatures, but the prediction awaits confirmation.

Note that a similar distinction between dynamic and thermodynamic quantities as drawn here, allows to understand non-monotonic behavior in the crystal growth velocity of glass-forming melts ${ }^{63}$, as well as generic features of the pressure- versus temperature-dependence of the glass transition 64 .

MCT attributes slow kinetics to the caging of particles by their neighbors in the dense melt. Diffusion is then governed by the collective breaking of such cages corresponding to the slow relaxation of density fluctuations (called $\alpha$ relaxation or structural relaxation in the glasstransition literature). Since slow structural relaxation governs all mass-transport processes close to $T_{c}$, also the Onsager coefficient $L$ for interdiffusion couples strongly to the self-diffusion coefficients $D_{\alpha}^{s}$ of the individual species. In consequence, the Darken relation expressing $L$ through the $D_{\alpha}^{s}$ is well fulfilled. For structural-relaxation effects to dominate, the diffusion processes have to be sufficiently slow, say, $D<10^{-9} \mathrm{~m}^{2} / \mathrm{s}$. At higher temperatures, short-time correlated binary-collision dynamics as expressed through the Enskog theory of liquids becomes important; typically, for a binary mixture of species A and $\mathrm{B}$, this will mean that $\mathrm{A}-\mathrm{B}$ cross terms contribute significantly to the dynamics. In this case, the Darken equation will be violated, expressed by a correction factor $S$ significantly different from unity.

In the MD simulation, the strongest deviations from Darken's equation are found indeed in the concentration range around $x \approx 0.5$ and at high temperatures. For the temperatures discussed here, the worst error made by the Darken equation is around 66\%. In this region, Darken's assumption is qualitatively wrong, in the sense that the true Onsager coefficient can be smaller than either selfdiffusion coefficients. The error of the Darken equation diminishes upon lowering temperature, at the same time when the dynamic suppression of interdiffusion becomes dominant over its thermodynamic enhancement.

Qualitative features of the kinetic mass transport processes in dense metallic melts can often be described by a hard-sphere analogy $\sqrt{5}$, emphasizing their entropic nature. As predicted by MCT, the slowing down upon mixing is a generic effect for mixtures composed of similar-sized spheres. A qualitative assessment of the melt's diffusion dynamics based on experimental data for the density, in terms of an effective hard-sphere packing fraction and size ratio, indeed gives reasonable results for $\mathrm{Al}-\mathrm{Ni}$, but slightly less so for Zr-Ni (which may indicate stronger contributions from chemical short-range order ${ }^{32}$ ). The strong thermodynamic driving forces seen in the interdiffusion in metallic melts, on the other hand, are a nonentropic effect that is only poorly captured by the hardsphere analogy.

Our MD simulations intend to capture typical dynamical processes that arise in metallic melts in addition to the entropic effects caused by strong excluded-volume interactions. When performing quantitative comparisons, one has, however, to be aware that the modeling in terms of effective interaction potentials, such as the embeddedatom potentials used here, has its limitations. We have based our discussion on effective potential models that are gauged against the best available experimental data for the dynamics in the dense melt, rather than against crystallographic data (which is traditionally the case). Further experiments, for example on interdiffusion coefficients, will be needed to judge the quality of the MD models.

The results discussed here for the thermodynamic factor $\Phi$ and the correction factor $S$, are expected to be generically valid in mixing systems, where chemical ordering induces quantitative deviations from the Darken equation, leading to $S \lesssim 1$ typically 34 . Note that in systems exhibiting liquid-liquid demixing, the behavior close to this phase transition will be quite different: at the critical point of this phase transition, $S$ diverges, but $\Phi$ approaches zero more rapidly and the interdiffusion coefficient vanishes 2265 . For melts where precursors of the vicinity to such a phase transition are important, additional generic mixing phenomena (connected to the fact that $\Phi<1$, as, e.g., in $\mathrm{Ag}-\mathrm{Cu}$ ) will arise that are not discussed in the present paper. 


\section{ACKNOWLEDGMENTS}

We thank D. Holland-Moritz and J. Brillo for discussion. Part of this work was supported by the Helmholtz-
Gemeinschaft, Helmholtz-Hochschul-Nachwuchsgruppe VH-NG 406.
${ }^{1}$ F. Faupel, W. Frank, M.-P. Macht, H. Mehrer, V. Naundort, K. Rätzke, H. R. Schober, S. K. Sharma, and H. Teichler, Rev. Mod. Phys. 75, 237 (2003).

2 G. Kasperovich, A. Meyer, and L. Ratke, Int. Foundry Res. 62(4), 8 (2010).

3 J.-P. Boon and S. Yip, Molecular Hydrodynamics (McGraw Hill, New York, 1980).

4 W. Götze, Complex Dynamics of Glass-Forming Liquids (Oxford University Press, 2009).

5 A. Meyer, Phys. Rev. B 66, 134205 (2002).

6 J. Brillo, A. I. Pommrich, and A. Meyer, Phys. Rev. Lett. 107, 165902 (2011).

7 M. Shimoji and T. Itami, Defect and Diffusion Forum 43, 154 (1986).

8 A. Griesche, M.-P. Macht, J.-P. Garandet, and G. Frohberg, J. Non-Cryst. Solids 336, 173 (2004).

9 B. Zhang, A. Griesche, and A. Meyer, Phys. Rev. Lett. 104, 035902 (2010).

${ }^{10}$ F. Kargl, M. Engelhardt, F. Yang, H. Weis, P. Schmakat, B. Schillinger, A. Griesche, and A. Meyer, J. Phys.: Condens. Matter 23, 254201 (2011).

11 F. Kargl, E. Sondermann, H. Weis, and A. Meyer, High Temp. High Press. 42, 3 (2013).

12 J. P. Garandet, C. Barat, and T. Duffar, Int. J. Heat Mass Transf. 38, 2169 (1995); C. Barat and J. P. Garandet, ibid. 39, 2177 (1996).

${ }^{13}$ W. Sutherland, Phil. Mag. Ser. 6 9, 781 (1905).

14 C. De Michele and D. Leporini, Phys. Rev. E 63, 036701 (2001).

15 F. Fujara, B. Geil, H. Sillescu, and G. Fleischer, Z. Phys. B 88, 195 (1992).

16 M. D. Ediger, Annu. Rev. Phys. Chem. 51, 99 (2000).

17 R. Zwanzig and A. K. Harrison, J. Chem. Phys. 83, 5861 (1985).

18 A. M. Puertas and Th. Voigtmann, "Microrheology of colloidal systems," (2014), submitted.

19 L. S. Darken, Trans. AIME 175, 184 (1948).

20 J. Horbach, S. K. Das, A. Griesche, M.-P. Macht, G. Frohberg, and A. Meyer, Phys. Rev. B 75, 174304 (2007).

21 J.-P. Hansen and I. R. McDonald, Theory of Simple Liquids (Academic Press, London, 1986).

22 J. R. Manning, Phys. Rev. 124, 470 (1961).

${ }^{23}$ H. Ehmler, A. Heesemann, K. Rätzke, F. Faupel, and U. Geyer, Phys. Rev. Lett. 80, 4919 (1998).

${ }^{24}$ V. Zöllmer, K. Rätzke, F. Faupel, and A. Meyer, Phys. Rev. Lett. 90, 195502 (2003).

25 A. Bartsch, K. Rätzke, F. Faupel, and A. Meyer, Appl. Phys. Lett. 89, 121917 (2006).

26 A. Bartsch, K. Rätzke, A. Meyer, and F. Faupel, Phys. Rev. Lett. 104, 195901 (2010).

27 R. J. Baxter, J. Chem. Phys. 52, 4559 (1970).

28 J.-L. Barrat and A. Latz, J. Phys.: Condens. Matter 2, 4289 (1990).

${ }^{29}$ W. Götze and Th. Voigtmann, Phys. Rev. E 67, 021502 (2003).
30 G. Foffi, W. Götze, F. Sciortino, P. Tartaglia, and Th. Voigtmann, Phys. Rev. Lett. 91, 085701 (2003).

31 G. Foffi, W. Götze, F. Sciortino, P. Tartaglia, and Th. Voigtmann, Phys. Rev. E 69, 011505 (2004).

32 Th. Voigtmann, A. Meyer, D. Holland-Moritz, S. Stüber, T. Hansen, and T. Unruh, EPL 82, 66001 (2008).

33 S. K. Das, J. Horbach, M. M. Koza, S. Mavila Chatoth, and A. Meyer, Appl. Phys. Lett. 86, 011918 (2005).

34 A. Griesche, B. Zhang, J. Horbach, and A. Meyer, Def. Diff. Forum 289-292, 705 (2009).

35 S. Stüber, D. Holland-Moritz, T. Unruh, and A. Meyer, Phys. Rev. B 81, 024204 (2010).

36 Y. Mishin, M. J. Mehl, and D. A. Papaconstantopoulos, Phys. Rev. B 65, 224114 (2002).

37 A. Kerrache, J. Horbach, and K. Binder, EPL 81, 58001 (2008).

38 S. K. Das, J. Horbach, and Th. Voigtmann, Phys. Rev. B 78, 064208 (2008).

39 T. Kumagai, D. Nikkuni, S. Hara, S. Izumi, and S. Sakai, Mater. Trans. 48, 1313 (2007).

40 T. Kordel, D. Holland-Moritz, F. Yang, J. Peters, T. Unruh, T. Hansen, and A. Meyer, Phys. Rev. B 83, 104205 (2011).

41 H. Teichler, Phys. Rev. Lett. 76, 62 (1996).

42 A. B. Mutiara and H. Teichler, Phys. Rev. E 64, 046133 (2001).

43 I. Ladadwa and H. Teichler, Phys. Rev. E 73, 031501 (2006).

44 H. Teichler, Phys. Rev. Lett. 107, 067801 (2011).

45 F. Sciortino and W. Kob, Phys. Rev. Lett. 86, 648 (2001).

46 Th. Voigtmann and J. Horbach, Phys. Rev. Lett. 103, 205901 (2009).

47 Th. Voigtmann, EPL 96, 36006 (2011).

48 H. M. van Beijeren and M. H. Ernst, Physica 68, 437 (1973); 70, 225 (1973).

49 A. Meyer, S. Stüber, D. Holland-Moritz, O. Heinen, and T. Unruh, Phys. Rev. B 77, 092201 (2008).

50 J. P. Garandet, G. Mathiak, V. Botton, P. Lehmann, and A. Griesche, Int. J. Thermophys. 25, 249 (2004).

51 W. Huang and Y. A. Chang, Intermetallics 6, 487 (1998).

52 G. Ghosh, J. Mater. Res. 9, 598 (1994).

53 W. Y. Wang, H. Z. Fang, S. L. Shang, H. Zhang, Y. Wang, X. Hui, S. Mathaudhu, and Z. K. Liu, Physica B: Cond. Matt. 406, 3089 (2011).

54 E. Sondermann et al., (2014), to be published.

55 D. Holland-Moritz, S. Stüber, H. Hartmann, T. Unruh, and A. Meyer, J. Phys.: Conf. Ser. 144, 012119 (2009).

56 N. Karpe, J. P. Krog, J. Bøttiger, N. G. Chechenin, R. E. Somekh, and A. L. Greer, Acta Metall. Mater. 43, 551 (1995).

57 L. Pauling, J. Am. Chem. Soc. 69, 542 (1947).

58 B. Cordero, V. Gómez, A. E. Platero-Prats, M. Revés, J. Echeverría, E. Cremandes, F. Barragáan, and S. Alvarez, Dalton Trans. , 2832 (2008).

59 Y. Plevachuk, I. Egry, J. Brillo, D. Holland-Moritz, and 
I. Kaban, Int. J. Mater. Res. 98, 107 (2007).

60 P.-F. Paradis, T. Ishikawa, G.-W. Lee, D. Holland-Moritz, J. Brillo, W.-K. Rhim, and J. T. Okada, Mater. Sci. Engin. R 76, 1 (2014).

61 R. H. Davies, A. T. Dinsdale, J. A. Gisby, J. A. J. Robinson, and S. M. Martin, CALPHAD 26, 229 (2002), http://resource.npl.co.uk/mtdata/databases.htm.

62 Y. Q. Cheng and E. Ma, Prog. Mater. Sci. 56, 379 (2011).

${ }^{63}$ H. Wang, D. M. Herlach, and R. P. Liu, EPL 105, 36001 (2014).

64 Th. Voigtmann, Phys. Rev. Lett. 101, 095701 (2008).

${ }^{65}$ S. K. Das, M. E. Fisher, J. V. Sengers, J. Horbach, and K. Binder, Phys. Rev. Lett. 97, 025702 (2006). 